\title{
Extended Ellipsoidal Outer-Bounding Set-Membership Estimation for Nonlinear Discrete-Time Systems with Unknown-but-Bounded Disturbances
}

\author{
Yushuang Liu, Yan Zhao, and Falin Wu \\ School of Instrumentation Science and Opto-Electronics Engineering, Beihang University (BUAA), Beijing 100191, China \\ Correspondence should be addressed to Yushuang Liu; ysliu@buaa.edu.cn and Falin Wu; falin.wu@buaa.edu.cn
}

Received 15 February 2016; Accepted 5 April 2016

Academic Editor: Driss Boutat

Copyright (c) 2016 Yushuang Liu et al. This is an open access article distributed under the Creative Commons Attribution License, which permits unrestricted use, distribution, and reproduction in any medium, provided the original work is properly cited.

\begin{abstract}
This paper develops an extended ellipsoidal outer-bounding set-membership estimation (EEOB-SME) algorithm with high accuracy and efficiency for nonlinear discrete-time systems under unknown-but-bounded (UBB) disturbances. The EEOB-SME linearizes the first-order terms about the current state estimations and bounds the linearization errors by ellipsoids using interval analysis for nonlinear equations of process and measurement equations, respectively. It has been demonstrated that the EEOB-SME algorithm is stable and the estimation errors of the EEOB-SME are bounded when the nonlinear system is observable. The EEOBSME decreases the computation load and the feasible sets of EEOB-SME contain more true states. The efficiency of the EEOB-SME algorithm has been shown by a numerical simulation under UBB disturbances.
\end{abstract}

\section{Introduction}

State estimation of dynamical systems from disturbances observations is one of the fundamental problems in control and signal processing. Based on the Bayesian theory, traditional state estimation algorithms are commonly solved under statistics assumptions of process and measurement disturbances, which have been extensively studied in last decades. The most common approach in recursive estimation is Kalman Filter (KF) [1], and the corresponding stochastic state estimators are the extended Kalman Filter (EKF) [2, 3], unscented Kalman Filter [4], and particle filter [5] for nonlinear systems. However, traditional state estimation algorithms, which aim to construct the posterior density of the estimated state, estimates of the variance with stochastic disturbances under the stochastic framework, sometimes give biased results when statistical assumptions are not satisfied. In many real-time engineering applications, the probabilistic distribution assumptions of the process or measurement disturbances are often insufficient and cannot be determined precisely with a few system input and output records.
Under the assumption of unknown-but-bounded (UBB) disturbances, the set-membership estimation (SME) algorithm is considered to be an attractive alternative for state estimation [6]. Set-membership methods with deterministic noise assumptions have been largely investigated in the past 40 years in systems state or parameter estimation literatures. Unlike probabilistic approaches, the only assumptions of process and measurement disturbances are UBB and there are not any assumptions in the deterministic bounded disturbances. The solution of the set-membership estimation is a feasible set rather than the posterior density of state variables. The feasible set is a complex convex set in the state space, which is consistent with the system model, the input and output data, and UBB disturbances. The centre of the feasible set is used as a point estimate. Because of the complicated shape of the true feasible set, the key problem in setmembership estimation is to use simple convex geometric set, such as ellipsoid [7], interval [8], and zonotope [9], to reduce the complexity of algorithms. As the intuitive mathematical derivation and computational efficient algorithm, ellipsoidal set methods are claimed to be much better in many situations. 
The first ellipsoidal set method for set-membership state estimation was established in [6] and developed to optimal control problem with parametric families of ellipsoids in [10]. The optimal bounding ellipsoid for parameter identification is proposed in [11]. Under the minimum-volume and minimum-trace criteria, optimal ellipsoidal state-bounding algorithms were introduced in [12-14]. With selective measurement update scheme [15], a set-membership state estimation algorithm was proposed by minimizing the upper bound on a Lyapunov function of the estimation error in observation update [16] and was developed with the nonincreasing property of the estimation errors in [17]. The multi-input multioutput situation was developed in [18]. By maximizing the decrease of a Lyapunov function of the estimation error in the worse noise case, an input-to-state stable (ISS) set-membership state estimation was presented in [19]. Recently, with lower computation load than the setmembership state estimation algorithm in [19], the ellipsoidal state-bounding-based set-membership estimation (ES-SME) is proposed [20].

There are relatively fewer results to be available to deal with nonlinear cases for SME algorithms. A set-valued observer for nonlinear systems was produced in [21]. An extended set-membership filter for dynamic nonlinear systems was proposed with interval analysis and the convergence of the filter was proved in [22]. A UD factorization-based set-membership filter with suboptimal bounding ellipsoids to improve the numeric stability was presented in [23]. Based on DC programming, a set-membership state estimation algorithm for nonlinear discrete-time systems with tight bounds was developed in [24]. Using the Takagi-Sugeno fuzzy model, the set-membership fuzzy filtering was established for nonlinear discrete-time systems [25]. The ellipsoidal statebounding algorithms for nonlinear systems were used in flight control [26], neural networks training [27], tracking [28], and power system dynamic state estimation [29].

For nonlinear systems, the extended Kalman filter linearizes the state trajectory about the current state estimate and the EKF is known to give biased estimates. Using similar linearization technologies to EKF, nonlinear setmembership filters had been explored with predetermined explicit assumptions of linearization error bounds in [22, 23]. Due to the complexity of nonlinear transformation of feasible sets and the lack of accurate bounding information, the nonlinearity of the system may seriously degrade the performance of the above mentioned state estimation algorithms. There are still several challenges to estimate the state for nonlinear system with UBB disturbances.

To improve the estimation accuracy and reduce the computation load, this paper extends the ES-SME algorithm to nonlinear discrete-time systems and proposes an extended ellipsoidal outer-bounding set-membership estimation (EEOB-SME) algorithm. The paper is presented as follows. The ES-SME algorithm is introduced in Section 2. In Section 3, the ES-SME is extended to nonlinear system with interval analysis and the EEOB-SME algorithm is proposed. Section 4 analyses the stability of the proposed EEOB-SME. The effectiveness of the EEOB-SME is demonstrated through a numerical simulation in Section 5. Section 6 concludes the paper.

\section{Ellipsoidal Outer-Bounding SME for Linear System}

\subsection{Recursive Structure of the ES-SME}

Definition 1. An ellipsoid set is given by the set $E\left(\mathbf{a}, \sigma^{2} \mathbf{P}\right)=$ $\left\{\mathbf{x} \in \mathbf{R}^{n}:(\mathbf{x}-\mathbf{a})^{T}\left(\sigma^{2} \mathbf{P}\right)^{-1}(\mathbf{x}-\mathbf{a}) \leq 1\right\}$, where $\mathbf{a} \in \mathbf{R}^{n}$ is the centre of the ellipsoid and $\sigma^{2} \mathbf{P} \in \mathbf{R}^{n \times n}$ called the shape-defining matrix of ellipsoid is a symmetric positive definite matrix which defines the shape and orientation of the ellipsoid. The variable $\sigma^{2} \in \mathbf{R}$ is a scalar variable.

Consider the linear discrete-time system in state space form

$$
\begin{aligned}
& \mathbf{x}_{k}=\mathbf{F}_{k-1} \mathbf{x}_{k-1}+\mathbf{w}_{k-1}, \\
& \mathbf{y}_{k}=\mathbf{H}_{k} \mathbf{x}_{k}+\mathbf{v}_{k},
\end{aligned}
$$

where $\mathbf{x}_{k} \in \mathbf{R}^{n}$ is the state vector and $\mathbf{y}_{k} \in \mathbf{R}^{m}$ is the observation vector at time $k . \mathbf{F}_{k-1} \in \mathbf{R}^{n \times n}$ and $\mathbf{H}_{k} \in \mathbf{R}^{n \times m}$ are known matrices. The disturbance $\mathbf{w}_{k}$ and $\mathbf{v}_{k}$ are UBB disturbances in the time and measurement equations, which are confined to the following ellipsoid sets:

$$
\begin{aligned}
& \mathbf{w}_{k-1} \in E\left(0, \mathbf{W}_{k-1}\right) \Longleftrightarrow\left\{\mathbf{w}_{k-1} \in \mathbf{R}^{n}: \mathbf{w}_{k-1}^{T} \mathbf{W}_{k-1}^{-1} \mathbf{w}_{k-1} \leq 1\right\}, \\
& \mathbf{v}_{k} \in E\left(0, \mathbf{v}_{k}\right) \Longleftrightarrow\left\{\mathbf{v}_{k} \in \mathbf{R}^{m}: \mathbf{v}_{k}^{T} \mathbf{V}_{k}^{-1} \mathbf{v}_{k} \leq 1\right\},
\end{aligned}
$$

where $\mathbf{W}_{k-1}$ and $\mathbf{V}_{k}$ are known positive definite matrices. It is assumed that the initial state belongs to a known ellipsoid set

$$
\begin{aligned}
& E\left(0, \sigma_{0}^{2} \mathbf{P}_{0}\right) \\
& \quad=\left\{\mathbf{x}_{0} \in \mathbf{R}^{n}:\left(\mathbf{x}_{0}-\widehat{\mathbf{x}}_{0}\right)^{T} \mathbf{P}_{0}^{-1}\left(\mathbf{x}_{0}-\widehat{\mathbf{x}}_{0}\right) \leq \sigma_{0}^{2}\right\},
\end{aligned}
$$

where $\widehat{\mathbf{x}}_{0}$ is the estimation result of $\mathbf{x}_{0}$ at the initial time.

The recursive structures of ellipsoidal outer-bounding set-membership estimation are time update and measurement update, which are similar to the Kalman Filter. Assume at time $k-1$, the ellipsoid $E\left(\widehat{\mathbf{x}}_{k-1}, \sigma_{k-1}^{2} \mathbf{P}_{k-1}\right)$ contains the state vector $\mathbf{x}_{k-1}$. From (1) and (3), the priori state estimation $\mathbf{x}_{k \mid k-1}$ lies in the vector sum of the linear transform ellipsoid $\mathbf{F}_{k-1} E\left(\widehat{\mathbf{x}}_{k-1}, \sigma_{k-1}^{2} \mathbf{P}_{k-1}\right)$ and ellipsoid $E\left(0, \mathbf{W}_{k-1}\right)$,

$$
\mathbf{x}_{k \mid k-1} \in \mathbf{F}_{k-1} E\left(\widehat{\mathbf{x}}_{k-1}, \sigma_{k-1}^{2} \mathbf{P}_{k-1}\right) \oplus E\left(0, \mathbf{W}_{k-1}\right) .
$$

Generally, the vector sum of two ellipsoids is not an ellipsoid; we find an ellipsoid $E\left(\widehat{\mathbf{x}}_{k \mid k-1}, \sigma_{k \mid k-1}^{2} \mathbf{P}_{k \mid k-1}\right)$ called the state prediction ellipsoid to contain the convex set of the vector sum,

$$
\begin{aligned}
& E\left(\widehat{\mathbf{x}}_{k \mid k-1}, \sigma_{k \mid k-1}^{2} \mathbf{P}_{k \mid k-1}\right) \\
& \quad \supset \mathbf{F}_{k-1} E\left(\widehat{\mathbf{x}}_{k-1}, \sigma_{k-1}^{2} \mathbf{P}_{k-1}\right) \oplus E\left(0, \mathbf{W}_{k-1}\right) .
\end{aligned}
$$

At time $k$, the state $\mathbf{x}_{k}$ lies in the intersection of the observation ellipsoid $S_{k}$ and the state prediction ellipsoid $E\left(\widehat{\mathbf{x}}_{k \mid k-1}, \sigma_{k \mid k-1}^{2} \mathbf{P}_{k \mid k-1}\right)$,

$$
\mathbf{x}_{k} \in E\left(\widehat{\mathbf{x}}_{k \mid k-1}, \sigma_{k \mid k-1}^{2} \mathbf{P}_{k \mid k-1}\right) \cap S_{k},
$$


where the observation ellipsoid is

$$
S_{k}=\left\{\mathbf{x}_{k} \in \mathbf{R}^{n}:\left(\mathbf{y}_{k}-\mathbf{H}_{k} \mathbf{x}_{k}\right)^{T} \mathbf{V}_{k}^{-1}\left(\mathbf{y}_{k}-\mathbf{H}_{k} \mathbf{x}_{k}\right) \leq 1\right\} .
$$

Normally, the intersection set of two ellipsoids is not an ellipsoid. It has to find an ellipsoid $\mathbf{x}_{k} \in E\left(\widehat{\mathbf{x}}_{k}, \sigma_{k}^{2} \mathbf{P}_{k}\right)$ called the updated state ellipsoid to contain the intersection set,

$$
E\left(\widehat{\mathbf{x}}_{k}, \sigma_{k}^{2} \mathbf{P}_{k}\right) \supset E\left(\widehat{\mathbf{x}}_{k \mid k-1}, \sigma_{k \mid k-1}^{2} \mathbf{P}_{k \mid k-1}\right) \cap S_{k} .
$$

Lemma 2 (see [20]). The recursive procedures for ES-SME algorithm are the following equations.

Time Update. Assuming a known state $\mathbf{x}_{k-1}$ which lies in the ellipsoid $E\left(\widehat{\mathbf{x}}_{k-1}, \sigma_{k-1}^{2} \mathbf{P}_{k-1}\right)$, we have $\mathbf{x}_{k \mid k-1} \in E\left(\widehat{\mathbf{x}}_{k \mid k-1}\right.$, $\left.\sigma_{k \mid k-1}^{2} \mathbf{P}_{k \mid k-1}\right)$,

$$
\begin{aligned}
\widehat{\mathbf{x}}_{k \mid k-1}= & \mathbf{F}_{k-1} \widehat{\mathbf{x}}_{k-1}, \\
\mathbf{P}_{k \mid k-1}= & \left(1-\rho_{k}\right)^{-1} \mathbf{F}_{k-1} \mathbf{P}_{k-1} \mathbf{F}_{k-1}^{T} \\
& +\left(\sigma_{k \mid k-1}^{2} \rho_{k}\right)^{-1} \mathbf{W}_{k-1}, \\
\sigma_{k \mid k-1}^{2}= & \sigma_{k-1}^{2},
\end{aligned}
$$

where the value of parameter $\rho_{k}$ belongs to $(0,1)$ and $\sigma_{k \mid k-1}^{2}$ is positive.

Observation Update. Given the observation ellipsoid $S_{k}$ and the state prediction ellipsoid $E\left(\widehat{\mathbf{x}}_{k \mid k-1}, \sigma_{k \mid k-1}^{2} \mathbf{P}_{k \mid k-1}\right)$, the state $\mathbf{x}_{k} \in E\left(\widehat{\mathbf{x}}_{k}, \sigma_{k}^{2} \mathbf{P}_{k}\right)$,

$$
\begin{aligned}
\widehat{\mathbf{x}}_{k} & =\widehat{\mathbf{x}}_{k \mid k-1}+\mathbf{K}_{k} \boldsymbol{\delta}_{k}, \\
\mathbf{P}_{k} & =\frac{1}{1-\lambda_{k}}\left[\mathbf{I}-\mathbf{K}_{k} \mathbf{H}_{k}\right] \mathbf{P}_{k \mid k-1}, \\
\sigma_{k}^{2} & =\left(1-\lambda_{k}\right) \sigma_{k \mid k-1}^{2}+\lambda_{k}-\boldsymbol{\delta}_{k}^{T} \mathbf{Q}_{k}^{-1} \boldsymbol{\delta}_{k}, \\
\mathbf{K}_{k} & =\frac{1}{1-\lambda_{k}} \mathbf{P}_{k \mid k-1} \mathbf{H}_{k}^{T} \mathbf{Q}_{k}^{-1}, \\
\mathbf{Q}_{k} & =\frac{1}{\lambda_{k}} \mathbf{V}_{k}+\frac{1}{1-\lambda_{k}} \mathbf{H}_{k} \mathbf{P}_{k \mid k-1} \mathbf{H}_{k}^{T}, \\
\boldsymbol{\delta}_{k} & =\mathbf{y}_{k}-\mathbf{H}_{k} \widehat{\mathbf{x}}_{k \mid k-1},
\end{aligned}
$$

where the value of parameter $\lambda_{k}$ belongs to $(0,1)$ and $\sigma_{k}^{2}$ is positive.

Remark 3. The covariance of the posterior estimation error in the ES-SME algorithm can be written

$$
\begin{aligned}
\mathbf{P}_{k}= & \frac{1}{1-\lambda_{k}}\left[\mathbf{I}-\mathbf{K}_{k} \mathbf{H}_{k}\right] \mathbf{P}_{k \mid k-1}\left[\mathbf{I}-\mathbf{K}_{k} \mathbf{H}_{k}\right]^{T} \\
& +\frac{1}{\lambda_{k}} \mathbf{K}_{k} \mathbf{V}_{k} \mathbf{K}_{k}^{T} .
\end{aligned}
$$

And the gain matrix in the ES-SME algorithm has another expression

$$
\mathbf{K}_{k}=\lambda_{k} \mathbf{P}_{k} \mathbf{H}_{k}^{T} \mathbf{V}_{k}^{-1}
$$

Remark 4. The scalar variable $\sigma_{k}^{2}$ presents the upper bound of a Lyapunov function of the posteriori estimation error, which is the inequation $\left\|\mathbf{x}_{k}-\widehat{\mathbf{x}}_{k}\right\|_{\mathbf{P}_{k}^{-1}} \leq \sigma_{k}^{2}$.

Remark 5. Through the assumption of UBB disturbances, a time-varying ellipsoid set is acquired in state space which always contains the true state of linear system. The shapedefining matrices $\sigma_{k}^{2} \mathbf{P}_{k}$ of ellipsoids, which are equal to covariance matrices in KF, represent the uncertainty of the estimate results. The estimation accuracy is evaluated by the measure of the ellipsoid set. The size of an ellipsoid is measured by its volume or trace.

2.2. Optimal Parameters Selection in the ES-SME. Commonly, the minimum-volume or minimum-trace criterion is used to compute the optimal scalar parameters $\rho_{k}$ and $\lambda_{k}$ in time and measurement update. For the computation efficiency, parameter $\rho_{k}$ is computed under the minimumtrace criterion [14]

$$
\rho_{k}=\frac{\sqrt{\operatorname{tr}\left(\mathbf{W}_{k-1}\right)}}{\sqrt{\operatorname{tr}\left(\sigma_{k-1}^{2} \mathbf{F}_{k-1} \mathbf{P}_{k-1} \mathbf{F}_{k-1}^{T}\right)}+\sqrt{\operatorname{tr}\left(\mathbf{W}_{k-1}\right)}} .
$$

According to Lemma 6 , the optimal parameter $\lambda_{k}$ in measurement update is computed through minimizing the upper bound of scalar variable $\sigma_{k}^{2}$.

Lemma 6 (see [20]). Let the initial condition $\sigma_{0 \mid 0}^{2} \leq 1$ and $\beta_{k}=\left(1-\sigma_{k \mid k-1}^{2}\right) /\left(\overline{\boldsymbol{\delta}}_{k}^{T} \overline{\boldsymbol{\delta}}_{k}\right)$, where $\overline{\boldsymbol{\delta}}_{k}=\overline{\mathbf{V}}_{k} \boldsymbol{\delta}_{k}, \overline{\mathbf{G}}_{k}=$ $\overline{\mathbf{V}}_{k} \mathbf{H}_{k} \mathbf{P}_{k \mid k-1} \mathbf{H}_{k}^{T} \overline{\mathbf{V}}_{k}^{T}, \mathbf{V}_{k}^{-1}=\overline{\mathbf{V}}_{k}^{T} \overline{\mathbf{V}}_{k}$, and $\bar{g}_{k}$ is the maximum eigenvalue of $\overline{\mathbf{G}}_{k}$. Minimizing parameter $\lambda_{k}$ with respect to scalar variable $\bar{\sigma}_{k}^{2}$, where $\bar{\sigma}_{k}^{2}=\left(1-\lambda_{k}\right) \sigma_{k \mid k-1}^{2}+\lambda_{k}-\lambda_{k}(1-$ $\left.\lambda_{k}\right)\left(\overline{\boldsymbol{\delta}}_{k}^{T} \overline{\boldsymbol{\delta}}_{k} /\left(\left(1-\lambda_{k}\right)+\lambda_{k} \bar{g}_{k}\right)\right)$, one can obtain the following:

$$
\begin{aligned}
& \lambda_{k} \\
& = \begin{cases}0 & \sigma_{k \mid k-1}^{2}+\overline{\boldsymbol{\delta}}_{k}^{T} \overline{\boldsymbol{\delta}}_{k} \leq 1 \\
\frac{\left(1-\beta_{k}\right)}{2} & \bar{g}_{k}=1 \\
\frac{1}{1-\bar{g}_{k}}\left[1-\sqrt{\left.\frac{\bar{g}_{k}}{1+\beta_{k}\left(\bar{g}_{k}-1\right)}\right]}\right. & \bar{g}_{k} \neq 1,\end{cases}
\end{aligned}
$$

where parameter $\lambda_{k}$ lies in $[0,1)$.

Remark 7. The method in Lemma 6 avoids the complexity computation for minimum-volume or trace criterion [14] and avoids solving the equation with the same dimension of the output vector for Becis-Aubry's set-membership estimation (BA-SME) [19].

\section{Extended Ellipsoidal Outer-Bounding SME}

The EKF considers the first-order terms used for time update and measurement update, and the second-order terms is defined as the Lagrange remainder. The main idea of EEOB$\mathrm{SME}$ is to linearize the nonlinear systems about the current 
estimation and use interval mathematics to acquire the bound of the higher order terms. Then, the bound of the linearization error is combined together with the process or measurement disturbances to acquire a new disturbances ellipsoid, which can be seen as new UBB disturbances. The state prediction ellipsoid and the updated state ellipsoid can be estimated recursively by applying a linear set-membership estimation algorithm at each time step.

Consider a nonlinear discrete-time state space system

$$
\begin{aligned}
& \mathbf{x}_{k+1}=f\left(\mathbf{x}_{k}\right)+\mathbf{w}_{k}, \\
& \mathbf{y}_{k+1}=h\left(\mathbf{x}_{k+1}\right)+\mathbf{v}_{k+1},
\end{aligned}
$$

where $f(\cdot)$ and $h(\cdot)$ are nonlinear $C^{2}$ functions and the other variables are defined in Section 2.

Linearizing equation (24) about the current state estimate $\widehat{\mathbf{x}}_{k}$ yields

$$
\begin{aligned}
\mathbf{x}_{k+1}= & \left.f\left(\mathbf{x}_{k}\right)\right|_{\mathbf{x}_{k}=\widehat{\mathbf{x}}_{k}}+\left.\frac{\partial f\left(\mathbf{x}_{k}\right)}{\partial \mathbf{x}}\right|_{\mathbf{x}_{k}=\widehat{\mathbf{x}}_{k}}\left(\mathbf{x}_{k}-\widehat{\mathbf{x}}_{k}\right) \\
& +O\left(\mathbf{x}_{k}^{2}\right)+\mathbf{w}_{k},
\end{aligned}
$$

where $\partial f\left(\mathbf{x}_{k}\right) / \partial \mathbf{x}$ is the gradient of the nonlinear function $f(\cdot)$. The term $O\left(\mathbf{x}_{k}^{2}\right)$ represents the high order terms, which are equal to the linearization error.

Define an interval vector $\overline{\mathbf{X}}_{k}$ called the state interval bound, which can take on any value over the interval where $\left(\mathbf{x}_{k}-\widehat{\mathbf{x}}_{k}\right)$ is defined. Then the state interval bound $\overline{\mathbf{X}}_{k}$ based on the ellipsoid extrema is

$$
\overline{\mathbf{X}}_{k}^{i}=\left[\widehat{\mathbf{x}}_{k}^{i}-\sqrt{\mathbf{P}_{k}^{i, i}} \widehat{\mathbf{x}}_{k}^{i}+\sqrt{\mathbf{P}_{k}^{i, i}}\right], \quad i=1, \ldots, n,
$$

where the superscripts $i, j$ denote the $(i, j)$ element of a matrix [22].

Considering the scalar case for simplicity, (26) can be expanded with a remainder

$$
\begin{aligned}
\mathbf{x}_{k+1}= & \left.f\left(\mathbf{x}_{k}\right)\right|_{\mathbf{x}_{k}=\widehat{\mathbf{x}}_{k}}+\left.\frac{\partial f\left(\mathbf{x}_{k}\right)}{\partial \mathbf{x}}\right|_{\mathbf{x}_{k}=\widehat{\mathbf{x}}_{k}}\left(\mathbf{x}_{k}-\widehat{\mathbf{x}}_{k}\right)+\cdots \\
& +\left.\frac{f\left(\mathbf{x}_{k}\right)^{n_{r}}}{n_{r} !}\right|_{\mathbf{x}_{k}=\widehat{\mathbf{x}}_{k}}\left(\mathbf{x}_{k}-\widehat{\mathbf{x}}_{k}\right)^{n_{r}} \\
& +R_{n_{r}}\left(\mathbf{x}_{k}-\widehat{\mathbf{x}}_{k}, \overline{\mathbf{X}}_{k}\right)+\mathbf{w}_{k}
\end{aligned}
$$

where $R_{n_{r}}\left(\mathbf{x}_{k}-\widehat{\mathbf{x}}_{k}, \overline{\mathbf{X}}_{k}\right)$ is a remainder term and $f\left(\mathbf{x}_{k}\right)^{n_{r}}$ is the $\left(n_{r}\right)$ th derivative about the estimation $\mathbf{x}_{k}$. Evaluating $R_{n_{r}}\left(\mathbf{x}_{k}-\right.$ $\left.\widehat{\mathbf{x}}_{k}, \overline{\mathbf{X}}_{k}\right)$ by interval mathematics [30], $R_{n_{r}}\left(\mathbf{x}_{k}-\widehat{\mathbf{x}}_{k}, \overline{\mathbf{X}}_{k}\right)$ can be bounded by an interval, which is defined by the interval vector $\overline{\mathbf{X}}_{k}$. The interval of the Lagrange remainder is written as

$$
R_{n_{r}}\left(\mathbf{x}_{k}-\widehat{\mathbf{x}}_{k}, \overline{\mathbf{X}}_{k}\right)=\left.\frac{f\left(\overline{\mathbf{X}}_{k}\right)^{n_{r}+1}}{\left(n_{r}+1\right) !}\right|_{\mathbf{x}_{k}=\widehat{\mathbf{x}}_{k}}\left(\mathbf{x}_{k}-\widehat{\mathbf{x}}_{k}\right)^{n_{r}+1} .
$$

Considering the expansion of the dynamics (28) with $n_{r}=$ 1 , the remainder term can be seen as simple function of the Hessian of the nonlinear dynamics

$$
R_{2}\left(\mathbf{x}_{k}-\widehat{\mathbf{x}}_{k}, \overline{\mathbf{X}}_{k}\right)=\left.\frac{1}{2} \frac{\partial^{2} f\left(\overline{\mathbf{X}}_{k}\right)}{\partial \mathbf{x}^{2}}\right|_{\mathbf{x}_{k}=\widehat{\mathbf{x}}_{k}}\left(\mathbf{x}_{k}-\widehat{\mathbf{x}}_{k}\right)^{2}
$$

and (28) can be written as

$$
\begin{aligned}
\mathbf{x}_{k+1}= & \left.f\left(\mathbf{x}_{k}\right)\right|_{\mathbf{x}_{k}=\widehat{\mathbf{x}}_{k}}+\left.\frac{\partial f\left(\mathbf{x}_{k}\right)}{\partial \mathbf{x}}\right|_{\mathbf{x}_{k}=\widehat{\mathbf{x}}_{k}}\left(\mathbf{x}_{k}-\widehat{\mathbf{x}}_{k}\right) \\
& +\left.\frac{1}{2} \frac{\partial^{2} f\left(\overline{\mathbf{X}}_{k}\right)}{\partial \mathbf{x}^{2}}\right|_{\mathbf{x}_{k}=\widehat{\mathbf{x}}_{k}}\left(\mathbf{x}_{k}-\widehat{\mathbf{x}}_{k}\right)^{2}+\mathbf{w}_{k} .
\end{aligned}
$$

The expression for the general multistate case is shown

$$
\overline{\mathbf{X}}_{R_{2}}=\frac{1}{2} \operatorname{diag}\left(\overline{\mathbf{X}}_{k}-\widehat{\mathbf{x}}_{k}\right)^{T}\left[\begin{array}{c}
\mathbf{H}_{f^{1}}\left(\overline{\mathbf{X}}_{k}\right) \\
\vdots \\
\mathbf{H}_{f^{n}}\left(\overline{\mathbf{X}}_{k}\right)
\end{array}\right]\left(\overline{\mathbf{X}}_{k}-\widehat{\mathbf{x}}_{k}\right)
$$

where $\mathbf{H}_{f^{j}}(\cdot)=\partial^{2} f(\cdot) / \partial \mathbf{x}^{2}$ is the Hessian matrix of nonlinear function $f(\cdot)[22]$.

The interval of the Lagrange remainder can be bounded using an ellipsoid [12]. Minimizing the volume of the ellipsoid, a unique closed form solution is written as

$$
\left[\overline{\mathbf{W}}_{k}\right]^{i, j}= \begin{cases}2\left(\overline{\mathbf{X}}_{R_{2}}^{i+}-\overline{\mathbf{X}}_{R_{2}}^{i-}\right)^{2} & i=j \\ 0 & i \neq j,\end{cases}
$$

where the subscripts + and - denote the maximum and minimum values in the interval $\overline{\mathbf{X}}_{R_{k}}$. Then the ellipsoid of linearization error is defined as $E\left(0, \overline{\mathbf{W}}_{k}\right)$.

Combine with the process disturbance ellipsoid and the linearization error ellipsoid into a new ellipsoid bound called the extended process disturbance ellipsoid $E\left(0, \widehat{\mathbf{W}}_{k}\right)$

$$
\begin{aligned}
& \widehat{\mathbf{w}}_{k} \in E\left(0, \widehat{\mathbf{W}}_{k}\right) \supset E\left(0, \mathbf{W}_{k}\right) \oplus E\left(0, \overline{\mathbf{W}}_{k}\right) \\
& \widehat{\mathbf{W}}_{k}=\frac{\overline{\mathbf{W}}_{k}}{1-p_{\widehat{\mathbf{w}}_{k}}}+\frac{\mathbf{W}_{k}}{p_{\widehat{\mathbf{w}}_{k}}}
\end{aligned}
$$

where $p_{\widehat{\mathbf{w}}_{k}}$ is a parameter to be chosen to minimize the ellipsoid $E\left(0, \widehat{\mathbf{W}}_{k}\right)$. 
The measurement equation should be dealt with in the same way as calculating $\widehat{\mathbf{w}}_{k}$ to acquire the extended measurement disturbance ellipsoid $E\left(0, \widehat{\mathbf{V}}_{k+1}\right)$,

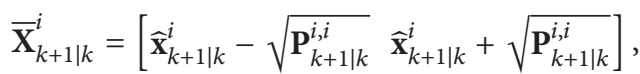

$$
\begin{aligned}
& i=1, \ldots, n \text {, } \\
& \overline{\mathbf{Y}}_{R_{2}}=R_{2}\left(\mathbf{x}_{k+1}-\widehat{\mathbf{x}}_{k+1 \mid k}, \overline{\mathbf{X}}_{k+1 \mid k}\right)=\frac{1}{2} \\
& \cdot \operatorname{diag}\left(\overline{\mathbf{X}}_{k+1 \mid k}-\widehat{\mathbf{x}}_{k+1 \mid k}\right)^{T} \\
& \cdot\left[\begin{array}{c}
\mathbf{H}_{h^{1}}\left(\overline{\mathbf{X}}_{k+1 \mid k}\right) \\
\vdots \\
\mathbf{H}_{h^{m}}\left(\overline{\mathbf{X}}_{k+1 \mid k}\right)
\end{array}\right]\left(\overline{\mathbf{X}}_{k}-\widehat{\mathbf{x}}_{k+1 \mid k}\right), \\
& {\left[\overline{\mathbf{V}}_{k+1}\right]^{i, j}= \begin{cases}2\left(\overline{\mathbf{Y}}_{R_{k}}^{i+}-\overline{\mathbf{Y}}_{R_{k}}^{i-}\right)^{2} & i=j \\
0 & i \neq j .\end{cases} } \\
& \widehat{\mathbf{v}}_{k+1} \in E\left(0, \widehat{\mathbf{V}}_{k+1}\right) \supset E\left(0, \mathbf{V}_{k+1}\right) \oplus E\left(0, \overline{\mathbf{V}}_{k+1}\right) \text {, } \\
& \widehat{\mathbf{V}}_{k+1}=\frac{\overline{\mathbf{V}}_{k+1}}{1-p_{\widehat{\mathbf{v}}_{k+1}}}+\frac{\mathbf{V}_{k+1}}{p_{\widehat{\mathbf{v}}_{k+1}}} .
\end{aligned}
$$

Remark 8. The specific procedures of the extended EOB-SME algorithm are summarized as follows:

(i) At time $k=1$, initialise $\widehat{\mathbf{x}}_{0}, \mathbf{P}_{0}$, and $\sigma_{0}^{2}=1$.

(ii) At each time step $k$, the state interval $\overline{\mathbf{X}}_{k}$ and the linearization error interval of process equation $\overline{\mathbf{X}}_{R_{2}}$ are given by (27) and (32). Then the extended process disturbance ellipsoid $E\left(0, \widehat{\mathbf{W}}_{k}\right)$ can be calculated by (35). Let $\widehat{\mathbf{x}}_{k+1 \mid k}=f\left(\widehat{\mathbf{x}}_{k}\right)$ and $\mathbf{F}_{k}=\left.\left(\partial f\left(\mathbf{x}_{k}\right) / \partial \mathbf{x}_{k}\right)\right|_{\mathbf{x}_{k}=\widehat{\mathbf{x}}_{k}}$; then the state prediction ellipsoid $E\left(\widehat{\mathbf{x}}_{k+1 \mid k}, \sigma_{k+1 \mid k}^{2} \mathbf{P}_{k+1 \mid k}\right)$ is computed by (11)-(13), where $\mathbf{W}_{k}$ is replaced by $\widehat{\mathbf{W}}_{k}$.

(iii) The extended measurement ellipsoid $E\left(0, \widehat{\mathbf{V}}_{k+1}\right)$ can be calculated by (36). Let $\widehat{\mathbf{y}}_{k+1}=h\left(\widehat{\mathbf{x}}_{k+1 \mid k}\right)$ and $\mathbf{H}_{k+1}=$ $\left.\left(\partial h\left(\mathbf{x}_{k+1}\right) / \partial \mathbf{x}_{k+1}\right)\right|_{\mathbf{x}_{k}=\widehat{\mathbf{x}}_{k+11 k}}$; then the updated ellipsoid $E\left(\widehat{\mathbf{x}}_{k+1}, \sigma_{k+1}^{2} \mathbf{P}_{k+1}\right)$ is given by (14)-(18), where $\mathbf{V}_{k+1}$ is replaced by $\widehat{\mathbf{V}}_{k+1}$ and the time index $k$ is changed to $k+1$. And $\boldsymbol{\delta}_{k+1}$ is $\boldsymbol{\delta}_{k+1}=\mathbf{y}_{k+1}-h\left(\widehat{\mathbf{x}}_{k+1 \mid k}\right)$.

(iv) Iterate recursive updates (i)-(iii) until the program is terminated.

\section{Stability Analysis}

In this section, the stability analysis of the EEOB-SME is presented based on analysing linearization error dynamics. Because the shape-defining matrix of ellipsoid is similar to the estimation error covariance matrix in KF, the way to the stability analysis is the following $[22,31]$. The EEOB-SME can be shown to be asymptotically stable with no disturbances and has an upper bound for the nonzero disturbances case.
Definition 9. The nonlinear observability test is satisfied if

$$
O\left(\widehat{\mathbf{x}}_{k}\right)=\left(\begin{array}{c}
\frac{\partial h}{\partial \mathbf{x}}\left(\mathbf{x}_{k}\right) \\
\frac{\partial h}{\partial \mathbf{x}}\left(\mathbf{x}_{k+1}\right) \frac{\partial f}{\partial \mathbf{x}}\left(\mathbf{x}_{k}\right) \\
\vdots \\
\frac{\partial h}{\partial \mathbf{x}}\left(\mathbf{x}_{k+n-1}\right) \frac{\partial f}{\partial \mathbf{x}}\left(\mathbf{x}_{k+n-2}\right) \cdots \frac{\partial f}{\partial \mathbf{x}}\left(\mathbf{x}_{k}\right)
\end{array}\right)
$$

has rank $n$.

Lemma 10 (see [32]). Consider the system given by (24) and (25) without process disturbances. Then there are $\exists \mu>0$ such that $\mathbf{F}_{k}=\partial f\left(\mathbf{x}_{k}\right) / \partial \mathbf{x}_{k}$ and $\mathbf{H}_{k+1}=\partial h\left(\mathbf{x}_{k+1}\right) / \partial \mathbf{x}_{k+1}$ satisfy the uniform observability test if $\left\|\mathbf{x}_{k}-\widehat{\mathbf{x}}_{k}\right\| \leq \mu$.

Lemma 11 (see [33]). If the pair $\left(\mathbf{F}_{k}, \mathbf{H}_{k}\right)$ is uniformly observable, then there exist two real numbers $\underline{s}$ and $\bar{s}$, such that $\underline{s} \times \mathbf{I} \leq$ $\mathbf{P}_{k+1 \mid k} \leq \bar{s} \times \mathbf{I}$ and $\underline{s} \times \mathbf{I} \leq \mathbf{P}_{k} \leq \bar{s} \times \mathbf{I}$.

Defining the estimation error

$$
\begin{gathered}
\zeta_{k}=\mathbf{x}_{k}-\widehat{\mathbf{x}}_{k \mid k-1}, \\
\zeta_{k+1}=\mathbf{x}_{k+1}-\widehat{\mathbf{x}}_{k+1 \mid k} .
\end{gathered}
$$

Let

$$
\begin{aligned}
f\left(\mathbf{x}_{k}\right)-f\left(\widehat{\mathbf{x}}_{k}\right) & =\mathbf{F}_{k}\left(\mathbf{x}_{k}-\widehat{\mathbf{x}}_{k}\right)+\varphi\left(\mathbf{x}_{k}, \widehat{\mathbf{x}}_{k}\right), \\
h\left(\mathbf{x}_{k}\right)-h\left(\widehat{\mathbf{x}}_{k \mid k-1}\right) & =\mathbf{H}_{k}\left(\mathbf{x}_{k}-\widehat{\mathbf{x}}_{k \mid k-1}\right)+\chi\left(\mathbf{x}_{k}, \widehat{\mathbf{x}}_{k \mid k-1}\right) .
\end{aligned}
$$

Then

$$
\begin{aligned}
\zeta_{k+1}= & f\left(\mathbf{x}_{k}\right)+\mathbf{w}_{k}-f\left(\widehat{\mathbf{x}}_{k}\right) \\
= & \mathbf{F}_{k}\left(\mathbf{x}_{k}-\widehat{\mathbf{x}}_{k}\right)+\varphi\left(\mathbf{x}_{k}, \widehat{\mathbf{x}}_{k}\right)+\mathbf{w}_{k} \\
= & \mathbf{F}_{k}\left(\mathbf{x}_{k}-\widehat{\mathbf{x}}_{k \mid k-1}\right)-\mathbf{F}_{k} \mathbf{K}_{k}\left(\mathbf{y}_{k}-h\left(\widehat{\mathbf{x}}_{k \mid k-1}\right)\right) \\
& +\varphi\left(\mathbf{x}_{k}, \widehat{\mathbf{x}}_{k}\right)+\mathbf{w}_{k} \\
= & \mathbf{F}_{k}\left(\mathbf{x}_{k}-\widehat{\mathbf{x}}_{k \mid k-1}\right) \\
& -\mathbf{F}_{k} \mathbf{K}_{k}\left(h\left(\mathbf{x}_{k}\right)+\mathbf{v}_{k}-h\left(\widehat{\mathbf{x}}_{k \mid k-1}\right)\right)+\varphi\left(\mathbf{x}_{k}, \widehat{\mathbf{x}}_{k}\right) \\
& +\mathbf{w}_{k} \\
= & \mathbf{F}_{k} \zeta_{k}-\mathbf{F}_{k} \mathbf{K}_{k} \mathbf{H}_{k}\left(\mathbf{x}_{k}-\widehat{\mathbf{x}}_{k \mid k-1}\right)+\varphi\left(\mathbf{x}_{k}, \widehat{\mathbf{x}}_{k}\right) \\
& -\mathbf{F}_{k} \mathbf{K}_{k} \chi\left(\mathbf{x}_{k}, \widehat{\mathbf{x}}_{k \mid k-1}\right)+\mathbf{w}_{k}-\mathbf{F}_{k} \mathbf{K}_{k} \mathbf{v}_{k} \\
= & \mathbf{F}_{k}\left(\mathbf{I}-\mathbf{K}_{k} \mathbf{H}_{k}\right) \zeta_{k}+\mathbf{r}_{k}+\mathbf{w}_{k}-\mathbf{F}_{k} \mathbf{K}_{k} \mathbf{v}_{k},
\end{aligned}
$$

where

$$
\mathbf{r}_{k}=\varphi\left(\mathbf{x}_{k}, \widehat{\mathbf{x}}_{k}\right)-\mathbf{F}_{k} \mathbf{K}_{k} \chi\left(\mathbf{x}_{k}, \widehat{\mathbf{x}}_{k \mid k-1}\right) .
$$

Theorem 12. Consider symmetric positive shape-defining matrices $\mathbf{P}_{k}$ and $\mathbf{P}_{k \mid k-1}$. Define $\Pi_{k}$ and $\Pi_{k \mid k-1}$ by $\Pi_{k \mid k-1}=$ 
$\mathbf{P}_{k \mid k-1}^{-1}$ and $\boldsymbol{\Pi}_{k}=\mathbf{P}_{k}^{-1}, p_{k+1}=\left(1-\rho_{k+1}\right) / \rho_{k+1}$, and assume $\mathbf{F}_{k}^{-1}$ and $\left(\mathbf{I}-\mathbf{K}_{k} \mathbf{H}_{k}\right)^{-T}$ exist for $k>0$. Then

$$
\begin{aligned}
& \boldsymbol{\Pi}_{k+1 \mid k} \leq\left(1+p_{k+1}^{-1}\right)^{-1}\left(1-\lambda_{k}\right) \mathbf{F}_{k}^{-T}\left(\mathbf{I}-\mathbf{K}_{k} \mathbf{H}_{k}\right)^{-T} \\
& \cdot\left[\boldsymbol{\Pi}_{k \mid k-1}-\left(1+p_{k+1}^{-1}\right)^{-1}\left(1-\lambda_{k}\right)\right. \\
& \cdot \boldsymbol{\Pi}_{k \mid k-1}\left(\left(1+p_{k+1}^{-1}\right)^{-1} \boldsymbol{\Pi}_{k}\right. \\
& \left.\left.+\sigma_{k}^{2}\left(1+p_{k+1}^{-1}\right)^{-1} \mathbf{F}_{k}^{T} \widehat{\mathbf{W}}_{k}^{-1} \mathbf{F}_{k}\right)^{-1} \boldsymbol{\Pi}_{k \mid k-1}\right](\mathbf{I} \\
& \left.\quad-\mathbf{K}_{k} \mathbf{H}_{k}\right)^{-1} \mathbf{F}_{k}^{-1} \cdot
\end{aligned}
$$

Proof. From (20), it is easy to obtain

$$
\mathbf{P}_{k} \geq \frac{1}{1-\lambda_{k}}\left[\mathbf{I}-\mathbf{K}_{k} \mathbf{H}_{k}\right] \mathbf{P}_{k \mid k-1}\left[\mathbf{I}-\mathbf{K}_{k} \mathbf{H}_{k}\right]^{T} .
$$

Taking the inverse of (44), we have

$$
\boldsymbol{\Pi}_{k} \leq\left(1-\lambda_{k}\right)\left[\mathbf{I}-\mathbf{K}_{k} \mathbf{H}_{k}\right]^{-T} \boldsymbol{\Pi}_{k \mid k-1}\left[\mathbf{I}-\mathbf{K}_{k} \mathbf{H}_{k}\right]^{-1} .
$$

From (12), we have

$$
\begin{aligned}
& \mathbf{P}_{k+1 \mid k} \\
& \quad=\mathbf{F}_{k}\left[\left(1+p_{k+1}^{-1}\right) \mathbf{P}_{k}+\frac{\left(1+p_{k+1}\right) \mathbf{F}_{k}^{-1} \mathbf{W}_{k} \mathbf{F}_{k}^{-T}}{\sigma_{k \mid k-1}^{2}}\right] \mathbf{F}_{k}^{T},
\end{aligned}
$$

and then

$$
\begin{aligned}
& \boldsymbol{\Pi}_{k+1 \mid k}=\mathbf{F}_{k}^{-T}\left[\left(1+p_{k+1}^{-1}\right) \mathbf{P}_{k}\right. \\
& \left.+\frac{\left(1+p_{k+1}\right) \mathbf{F}_{k}^{-1} \mathbf{W}_{k} \mathbf{F}_{k}^{-T}}{\sigma_{k}^{2}}\right]^{-1} \mathbf{F}_{k}^{-1}=\mathbf{F}_{k}^{-T}[(1 \\
& \left.\quad+p_{k+1}^{-1}\right)^{-1} \boldsymbol{\Pi}_{k}-\left(1+p_{k+1}^{-1}\right)^{-1} \Pi_{k}\left(\left(1+p_{k+1}^{-1}\right)^{-1} \boldsymbol{\Pi}_{k}\right. \\
& \left.\left.+\sigma_{k}^{2}\left(1+p_{k+1}\right)^{-1} \mathbf{F}_{k}^{T} \mathbf{W}_{k}^{-1} \mathbf{F}_{k}\right)^{-1}\left(1+p_{k+1}^{-1}\right)^{-1} \boldsymbol{\Pi}_{k}\right] \\
& \cdot \mathbf{F}_{k}^{-1} .
\end{aligned}
$$

From (15), it is obvious that

$$
\mathbf{P}_{k}^{T}=\frac{1}{1-\lambda_{k}} \mathbf{P}_{k \mid k-1}\left[\mathbf{I}-\mathbf{K}_{k} \mathbf{H}_{k}\right]^{T},
$$

and we obtain

$$
\begin{aligned}
\boldsymbol{\Pi}_{k} & =\left(1-\lambda_{k}\right) \boldsymbol{\Pi}_{k \mid k-1}\left[\mathbf{I}-\mathbf{K}_{k} \mathbf{H}_{k}\right]^{-1} \\
& =\left(1-\lambda_{k}\right)\left[\mathbf{I}-\mathbf{K}_{k} \mathbf{H}_{k}\right]^{-T} \boldsymbol{\Pi}_{k \mid k-1} .
\end{aligned}
$$

According to (45), we have

$$
\begin{aligned}
& \boldsymbol{\Pi}_{k+1 \mid k} \leq \mathbf{F}_{k}^{-T}\left[\left(1+p_{k+1}^{-1}\right)^{-1}\left(1-\lambda_{k}\right)\left(\mathbf{I}-\mathbf{K}_{k} \mathbf{H}_{k}\right)^{-T}\right. \\
& \cdot \boldsymbol{\Pi}_{k \mid k-1}\left(\mathbf{I}-\mathbf{K}_{k} \mathbf{H}_{k}\right)^{-1}-\left(1+p_{k+1}^{-1}\right)^{-1}\left(1-\lambda_{k}\right)(\mathbf{I} \\
& \left.-\mathbf{K}_{k} \mathbf{H}_{k}\right)^{-T} \boldsymbol{\Pi}_{k \mid k-1}\left(\left(1+p_{k+1}^{-1}\right)^{-1} \boldsymbol{\Pi}_{k}\right. \\
& \left.+\sigma_{k}^{2}\left(1+p_{k+1}\right)^{-1} \mathbf{F}_{k}^{T} \mathbf{W}_{k}^{-1} \mathbf{F}_{k}\right)^{-1}\left(1+p_{k+1}^{-1}\right)^{-1}(1 \\
& \left.\left.-\lambda_{k}\right) \boldsymbol{\Pi}_{k \mid k-1}\left(\mathbf{I}-\mathbf{K}_{k} \mathbf{H}_{k}\right)^{-1}\right] \mathbf{F}_{k}^{-1} \leq\left(1+p_{k+1}^{-1}\right)^{-1}(1 \\
& \left.-\lambda_{k}\right) \mathbf{F}_{k}^{-T}\left(\mathbf{I}-\mathbf{K}_{k} \mathbf{H}_{k}\right)^{-T}\left[\boldsymbol{\Pi}_{k \mid k-1}-\left(1+p_{k+1}^{-1}\right)^{-1}(1\right. \\
& \left.-\lambda_{k}\right) \boldsymbol{\Pi}_{k \mid k-1}\left(\left(1+p_{k+1}^{-1}\right)^{-1} \boldsymbol{\Pi}_{k}\right. \\
& \left.\left.+\sigma_{k}^{2}\left(1+p_{k+1}^{-1}\right)^{-1} \mathbf{F}_{k}^{T} \widehat{\mathbf{W}}_{k}^{-1} \mathbf{F}_{k}\right)^{-1} \boldsymbol{\Pi}_{k \mid k-1}\right](\mathbf{I} \\
& \left.-\mathbf{K}_{k} \mathbf{H}_{k}\right)^{-1} \mathbf{F}_{k}^{-1} .
\end{aligned}
$$

The proof of Theorem 12 is completed.

Theorem 13. Assume the following assumptions hold:

(i) $\left\|\mathbf{F}_{k}\right\| \leq \bar{a},\left\|\mathbf{H}_{k}\right\| \leq \bar{c},\left\|\mathbf{K}_{k}\right\| \leq \bar{k}, \underline{p} \times \mathbf{I} \leq \mathbf{P}_{k} \leq \bar{p} \times$ I, $\underline{p} \times \mathbf{I} \leq \mathbf{P}_{k \mid k-1} \leq \bar{p} \times \mathbf{I}$.

(ii) $\mathbf{F}_{k}$ is invertible for all $k \geq 0$.

(iii) There are positive real numbers $\varepsilon_{\varphi}, \varepsilon_{\chi}, \kappa_{\varphi}$, and $\kappa_{\chi}$, such that nonlinear functions $\varphi(\cdot)$ and $\chi(\cdot)$ in (39) and (40) are bounded as $\left\|\varphi\left(\mathbf{x}_{k}, \widehat{\mathbf{x}}_{k}\right)\right\| \leq \kappa_{\varphi}\left\|\mathbf{x}_{k}-\widehat{\mathbf{x}}_{k}\right\|^{2}$ and $\left\|\chi\left(\mathbf{x}_{k}, \widehat{\mathbf{x}}_{k \mid k-1}\right)\right\| \leq \kappa_{\chi}\left\|\mathbf{x}_{k}-\widehat{\mathbf{x}}_{k \mid k-1}\right\|^{2}$ for $\mathbf{x}_{k}, \widehat{\mathbf{x}}_{k}$, and $\widehat{\mathbf{x}}_{k \mid k-1} \in R^{n}$ with $\left\|\mathbf{x}_{k}-\widehat{\mathbf{x}}_{k}\right\| \leq \varepsilon_{\varphi}$ and $\left\|\mathbf{x}_{k}-\widehat{\mathbf{x}}_{k \mid k-1}\right\| \leq \varepsilon_{\chi}$, respectively.

Then the EEOB-SME algorithm gives ellipsoidal estimates where the error between the centre of the ellipsoid and the true state converges to zero, when the disturbances are zero.

Proof. From (14) and (40), $\mathbf{x}_{k}-\widehat{\mathbf{x}}_{k}=\mathbf{x}_{k}-\widehat{\mathbf{x}}_{k \mid k-1}-\mathbf{K}_{k} \mathbf{H}_{k}\left(\mathbf{x}_{k}-\right.$ $\left.\widehat{\mathbf{x}}_{k \mid k-1}\right)-\mathbf{K}_{k} \chi\left(\mathbf{x}_{k}, \widehat{\mathbf{x}}_{k \mid k-1}\right)-\mathbf{K}_{k} \mathbf{v}_{k}$. When the disturbances $\mathbf{v}_{k}$ are zero, we have

$$
\begin{aligned}
\mathbf{x}_{k}-\widehat{\mathbf{x}}_{k}= & \mathbf{x}_{k}-\widehat{\mathbf{x}}_{k \mid k-1}-\mathbf{K}_{k} \mathbf{H}_{k}\left(\mathbf{x}_{k}-\widehat{\mathbf{x}}_{k \mid k-1}\right) \\
& -\mathbf{K}_{k} \chi\left(\mathbf{x}_{k}, \widehat{\mathbf{x}}_{k \mid k-1}\right),
\end{aligned}
$$

According to the assumptions (i) and (iii) it is not difficult to see that

$$
\begin{aligned}
\left\|\mathbf{x}_{k}-\widehat{\mathbf{x}}_{k}\right\| \leq & \left\|\mathbf{x}_{k}-\widehat{\mathbf{x}}_{k \mid k-1}\right\|+\bar{k} \bar{c}\left\|\mathbf{x}_{k}-\widehat{\mathbf{x}}_{k \mid k-1}\right\| \\
& +\bar{k} \kappa_{\chi}\left\|\mathbf{x}_{k}-\widehat{\mathbf{x}}_{k \mid k-1}\right\|^{2} \\
\leq & \left(1+\bar{k} \bar{c}+\bar{k} \kappa_{\chi} \varepsilon_{\chi}\right)\left\|\mathbf{x}_{k}-\widehat{\mathbf{x}}_{k \mid k-1}\right\| .
\end{aligned}
$$


From (42), we obtain

$$
\left\|\mathbf{r}_{k}\right\| \leq\left\|\varphi\left(\mathbf{x}_{k}, \widehat{\mathbf{x}}_{k}\right)\right\|+\bar{a} \bar{k}\left\|\chi\left(\mathbf{x}_{k}, \widehat{\mathbf{x}}_{k \mid k-1}\right)\right\| .
$$

Choosing

$$
\varepsilon=\min \left(\varepsilon_{\chi}, \frac{\varepsilon_{\varphi}}{1+\bar{k} \bar{c}+\bar{k} \kappa_{\chi} \varepsilon_{\chi}}\right),
$$

for any $\left\|\mathbf{x}_{k}-\widehat{\mathbf{x}}_{k \mid k-1}\right\| \leq \varepsilon$, it is easy to verify that

$$
\begin{aligned}
\left\|\mathbf{r}_{k}\right\| \leq & \kappa_{\varphi}\left(1+\bar{k} \bar{c}+\bar{k} \kappa_{\chi} \varepsilon_{\chi}\right)\left\|\mathbf{x}_{k}-\widehat{\mathbf{x}}_{k \mid k-1}\right\|^{2} \\
& +\bar{a} \bar{k} \kappa_{\chi}\left\|\mathbf{x}_{k}-\widehat{\mathbf{x}}_{k \mid k-1}\right\|^{2} .
\end{aligned}
$$

Let $\kappa=\kappa_{\varphi}\left(1+\bar{k} \bar{c}+\bar{k} \kappa_{\chi} \varepsilon_{\chi}\right)+\bar{a} \bar{k} \kappa_{\chi}$; we have

$$
\left\|\mathbf{r}_{k}\right\| \leq \kappa\left\|\mathbf{x}_{k}-\widehat{\mathbf{x}}_{k \mid k-1}\right\|^{2}
$$

Define the Lyapunov function $\mathscr{V}_{k}\left(\zeta_{k}\right)=\boldsymbol{\zeta}_{k}^{T} \boldsymbol{\Pi}_{k \mid k-1} \zeta_{k} ;$ from the assumption (i), we obtain

$$
\frac{1}{\bar{p}}\left\|\zeta_{k}\right\|^{2} \leq \mathscr{V}_{k}\left(\zeta_{k}\right) \leq \frac{1}{\underline{p}}\left\|\zeta_{k}\right\|^{2} .
$$

When the disturbances are zero in (41), the estimation error is $\boldsymbol{\zeta}_{k+1}=\mathbf{F}_{k}\left(\mathbf{I}-\mathbf{K}_{k} \mathbf{H}_{k}\right) \boldsymbol{\zeta}_{k}+\mathbf{r}_{k}$ and the following can be obtained

$$
\begin{aligned}
& \mathscr{V}_{k+1}\left(\boldsymbol{\zeta}_{k+1}\right)=\boldsymbol{\zeta}_{k+1}^{T} \boldsymbol{\Pi}_{k+1 \mid k} \boldsymbol{\zeta}_{k+1}=\boldsymbol{\zeta}_{k}^{T}\left(\mathbf{I}-\mathbf{K}_{k} \mathbf{H}_{k}\right)^{T} \\
& \cdot \mathbf{F}_{k}^{T} \boldsymbol{\Pi}_{k+1 \mid k} \mathbf{F}_{k}\left(\mathbf{I}-\mathbf{K}_{k} \mathbf{H}_{k}\right)+2 \mathbf{r}_{k}^{T} \boldsymbol{\Pi}_{k+1 \mid k} \mathbf{F}_{k}(\mathbf{I} \\
& \left.-\mathbf{K}_{k} \mathbf{H}_{k}\right) \boldsymbol{\zeta}_{k}+\mathbf{r}_{k}^{T} \boldsymbol{\Pi}_{k+1 \mid k} \mathbf{r}_{k} \leq \boldsymbol{\zeta}_{k}^{T}\left(1+p_{k+1}^{-1}\right)^{-1}(1 \\
& \left.-\lambda_{k}\right)\left[\boldsymbol{\Pi}_{k \mid k-1}-\left(1+p_{k+1}^{-1}\right)^{-1}\left(1-\lambda_{k}\right)\right. \\
& \cdot \boldsymbol{\Pi}_{k \mid k-1}\left(\left(1+p_{k+1}^{-1}\right)^{-1} \boldsymbol{\Pi}_{k}\right. \\
& \left.\left.+\sigma_{k}^{2}\left(1+p_{k+1}\right)^{-1} \mathbf{F}_{k}^{T} \mathbf{W}_{k}^{-1} \mathbf{F}_{k}\right)^{-1} \boldsymbol{\Pi}_{k \mid k-1}\right] \boldsymbol{\zeta}_{k} \\
& +2 \mathbf{r}_{k}^{T} \boldsymbol{\Pi}_{k+1 \mid k} \mathbf{F}_{k}\left(\mathbf{I}-\mathbf{K}_{k} \mathbf{H}_{k}\right) \boldsymbol{\zeta}_{k}+\mathbf{r}_{k}^{T} \boldsymbol{\Pi}_{k+1 \mid k} \mathbf{r}_{k} .
\end{aligned}
$$

Then (58) can be written as

$$
\begin{aligned}
\mathscr{V}_{k+1}\left(\zeta_{k+1}\right) & \left(1+p_{k+1}^{-1}\right)^{-1}\left(1-\lambda_{k}\right) \zeta_{k}^{T} \Pi_{k \mid k-1} \zeta_{k} \\
\leq & -\frac{\left(1+p_{k+1}^{-1}\right)^{-1}\left(1-\lambda_{k}\right)^{2}\left\|\zeta_{k}\right\|^{2}}{\underline{p}^{2}\left[\left(1+p_{k+1}^{-1}\right)^{-1} \underline{p}+\sigma_{k}^{2}\left(1+p_{k+1}\right) \bar{a}^{2} / \underline{w}\right]} \\
+ & 2 \kappa\left\|\zeta_{k}\right\|^{2} \frac{\bar{a}(1+\bar{k} \bar{c})}{\underline{p}}\left\|\zeta_{k}\right\|+\kappa\left\|\zeta_{k}\right\|^{2} \frac{\kappa \varepsilon}{\underline{p}}\left\|\zeta_{k}\right\| .
\end{aligned}
$$

We obtain that

$$
\begin{gathered}
\mathscr{V}_{k+1}\left(\zeta_{k+1}\right)-\mathscr{V}_{k}\left(\zeta_{k}\right) \leq\left[\left(1+p_{k+1}^{-1}\right)^{-1}\left(1-\lambda_{k}\right)-1\right] \\
\cdot \mathscr{V}_{k+1}\left(\zeta_{k+1}\right) \\
-\left(\frac{\left(1+p_{k+1}^{-1}\right)^{-1}\left(1-\lambda_{k}\right)^{2}}{\underline{p}^{2}\left[\left(1+p_{k+1}^{-1}\right)^{-1} \underline{p}+\sigma_{k}^{2}\left(1+p_{k+1}\right) \bar{a}^{2} / \underline{w}\right]}\right.
\end{gathered}
$$

$$
\left.-\kappa^{\prime}\left\|\zeta_{k}\right\|\right)\left\|\zeta_{k}\right\|^{2}
$$

where $\kappa^{\prime}=\kappa[\bar{a}(1+\bar{k} \bar{c})+\kappa \varepsilon] / p$, because $\left[\left(1+p_{k+1}^{-1}\right)^{-1}\left(1-\lambda_{k}\right)-\right.$ 1] $=\left(-\lambda_{k}-p_{k+1}^{-1}\right) /\left(1+p_{k+1}^{-1}\right)<0$, choosing

$$
\begin{aligned}
& \varepsilon^{\prime}=\min (\varepsilon, \\
& \left.\frac{\left(1+p_{k+1}^{-1}\right)^{-1}\left(1-\lambda_{k}\right)^{2}}{\kappa^{\prime} \underline{p}^{2}\left[\left(1+p_{k+1}^{-1}\right)^{-1} \underline{p}+\sigma_{k}^{2}\left(1+p_{k+1}\right) \bar{a}^{2} / \underline{w}\right]}\right) .
\end{aligned}
$$

It follows that $\mathscr{V}_{k+1}\left(\zeta_{k+1}\right)-\mathscr{V}_{k}\left(\zeta_{k}\right) \leq 0$ holds for $\left\|\zeta_{k}\right\| \leq$ $\varepsilon^{\prime}$. Then the estimation error will go to zero. The proof of Theorem 13 is completed.

Theorem 14. Under the same assumptions in Theorem 13, the estimation error of EEOB-SME algorithm will acquire an upper bound, when the disturbances are nonzero.

Proof. When the disturbances are nonzero, the estimation error is described as

$$
\zeta_{k+1}=\mathbf{F}_{k}\left(\mathbf{I}-\mathbf{K}_{k} \mathbf{H}_{k}\right) \boldsymbol{\zeta}_{k}+\mathbf{r}_{k}+\mathbf{w}_{k}-\mathbf{F}_{k} \mathbf{K}_{k} \mathbf{v}_{k}
$$

Then the following can be obtained

$$
\begin{aligned}
& \mathscr{V}_{k+1}\left(\boldsymbol{\zeta}_{k+1}\right)=\boldsymbol{\zeta}_{k+1}^{T} \boldsymbol{\Pi}_{k+1 \mid k} \boldsymbol{\zeta}_{k+1} \leq \boldsymbol{\zeta}_{k}^{T}\left(1+p_{k+1}^{-1}\right)^{-1}(1 \\
& \left.-\lambda_{k}\right)\left[\boldsymbol{\Pi}_{k \mid k-1}-\left(1+p_{k+1}^{-1}\right)^{-1}\left(1-\lambda_{k}\right)\right. \\
& \cdot \boldsymbol{\Pi}_{k \mid k-1}\left(\left(1+p_{k+1}^{-1}\right)^{-1} \boldsymbol{\Pi}_{k}\right. \\
& \left.\left.\quad+\sigma_{k}^{2}\left(1+p_{k+1}\right)^{-1} \mathbf{F}_{k}^{T} \mathbf{W}_{k}^{-1} \mathbf{F}_{k}\right)^{-1} \boldsymbol{\Pi}_{k \mid k-1}\right] \boldsymbol{\zeta}_{k} \\
& \quad+2 \mathbf{r}_{k}^{T} \boldsymbol{\Pi}_{k+1 \mid k} \mathbf{F}_{k}\left(\mathbf{I}-\mathbf{K}_{k} \mathbf{H}_{k}\right) \boldsymbol{\zeta}_{k}+\mathbf{r}_{k}^{T} \boldsymbol{\Pi}_{k+1 \mid k} \mathbf{r}_{k} \\
& \quad+2 \mathbf{n}_{k}^{T} \boldsymbol{\Pi}_{k+1 \mid k} \mathbf{F}_{k}\left(\mathbf{I}-\mathbf{K}_{k} \mathbf{H}_{k}\right) \boldsymbol{\zeta}_{k}+\mathbf{n}_{k}^{T} \boldsymbol{\Pi}_{k+1 \mid k} \mathbf{n}_{k} \\
& \quad+2 \mathbf{n}_{k}^{T} \boldsymbol{\Pi}_{k+1 \mid k} \mathbf{r}_{k},
\end{aligned}
$$


where $\mathbf{n}_{k}=\mathbf{w}_{k}-\mathbf{F}_{k} \mathbf{K}_{k} \mathbf{v}_{k}$. The upper bound of disturbances is defined as $\bar{n}=\bar{w}-\bar{a} \bar{k} \bar{v}$. Thus we obtain

$$
\begin{aligned}
\mathscr{V}_{k+1}\left(\zeta_{k+1}\right) & \left(1+p_{k+1}^{-1}\right)^{-1}\left(1-\lambda_{k}\right) \zeta_{k}^{T} \Pi_{k \mid k-1} \zeta_{k} \\
& -\frac{\left(1+p_{k+1}^{-1}\right)^{-1}\left(1-\lambda_{k}\right)^{2}\left\|\zeta_{k}\right\|^{2}}{\underline{p}^{2}\left[\left(1+p_{k+1}^{-1}\right)^{-1} \underline{p}+\sigma_{k}^{2}\left(1+p_{k+1}\right) \bar{a}^{2} / \underline{w}\right]} \\
+ & 2 \kappa\left\|\zeta_{k}\right\|^{2} \frac{\bar{a}(1+\bar{k} \bar{c})}{\underline{p}}\left\|\zeta_{k}\right\|+\kappa\left\|\zeta_{k}\right\|^{2} \frac{\kappa \varepsilon}{\underline{p}}\left\|\zeta_{k}\right\| \\
& +\frac{2 \bar{n} \bar{a}(1+\bar{k} \bar{c})}{\underline{p}}\left\|\zeta_{k}\right\|+\frac{2 \bar{n} \kappa}{\underline{p}}\left\|\zeta_{k}\right\|^{2}+\frac{\bar{n}^{2}}{\underline{p}} .
\end{aligned}
$$

Then

$$
\begin{aligned}
& \mathscr{V}_{k+1}\left(\zeta_{k+1}\right)-\mathscr{V}_{k}\left(\zeta_{k}\right) \\
& \leq {\left[\left(1+p_{k+1}^{-1}\right)^{-1}\left(1-\lambda_{k}\right)-1\right] \mathscr{V}_{k+1}\left(\zeta_{k+1}\right) } \\
&+\kappa_{1}\left\|\zeta_{k}\right\|^{3}+\kappa_{2}\left\|\zeta_{k}\right\|^{2}+\kappa_{3}\left\|\zeta_{k}\right\|+\kappa_{4},
\end{aligned}
$$

where $\kappa_{i}$ and $\varepsilon_{i}$ are defined appropriately, and the following is obtained

$$
\begin{aligned}
& \mathscr{V}_{k+1}\left(\zeta_{k+1}\right)-\mathscr{V}_{k}\left(\zeta_{k}\right) \\
& \quad \leq\left[\left(1+p_{k+1}^{-1}\right)^{-1}\left(1-\lambda_{k}\right)-1\right] \mathscr{V}_{k+1}\left(\zeta_{k+1}\right)+\kappa_{4} .
\end{aligned}
$$

When $\left\|\zeta_{k}\right\| \geq \varepsilon_{4}$, the following holds: $\mathscr{V}_{k+1}\left(\boldsymbol{\zeta}_{k+1}\right)-\mathscr{V}_{k}\left(\boldsymbol{\zeta}_{k}\right) \leq$ 0 . It means estimation errors have an upper bound. The proof of Theorem 14 is completed.

\section{Numerical Simulation}

The following discrete-time Van der Pol equation within ellipsoidal bounds is to evaluate the performance of EEOBSME with EKF, BA-SME [19], and AESMF [23]:

$$
\begin{aligned}
{\left[\begin{array}{l}
\mathbf{x}_{1, k+1} \\
\mathbf{x}_{2, k+1}
\end{array}\right]=} & {\left[\begin{array}{c}
\mathbf{x}_{1, k}+h \mathbf{x}_{2, k} \\
\mathbf{x}_{2, k}+h\left(-9 \mathbf{x}_{1, k}+\mu\left(1-\mathbf{x}_{1, k}^{2}\right) \mathbf{x}_{2, k}\right)
\end{array}\right] } \\
& +\mathbf{w}_{k}, \\
\mathbf{y}_{k}= & \mathbf{x}_{1, k}+\mathbf{v}_{k},
\end{aligned}
$$

which was studied in [21]. The initial conditions are $h=$ $0.02, \mu=2, \mathbf{x}_{0}=(1,2)^{T}, \mathbf{P}_{0}=\operatorname{diag}(1,1)$, and $\sigma_{0}^{2}=1$. The process and measurement disturbances are uniformly distributed in $(-0.1,0.1)$ inside bounding ellipsoids, which have shape-defining matrix $\mathbf{W}=\operatorname{diag}(0.01,0.01)$ and $\mathbf{v}=$ $(-0.1,0.1)$. Simulation contains $M=100$ Monte Carlo steps with independent noise sequences and $N=200$ recursive updates in each Monte Carlo step. The geometric centre of ellipsoid is considered as the point estimation result and used the trace criterion to compute parameter $p_{k}$ in both SME

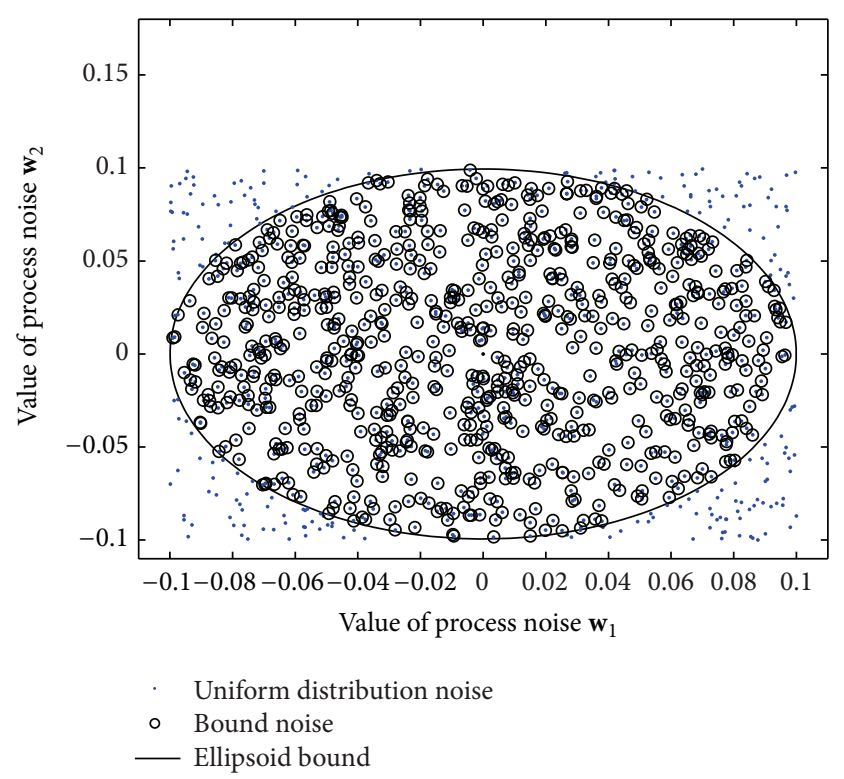

FIGURE 1: Uniformly distribution in ellipsoidal bound for process disturbances.

algorithms. The averaged root mean square error (RMSE) is defined as $\operatorname{RMSE}_{i}=\sqrt{\sum_{k=1}^{N}\left(\mathbf{x}_{k}-\widehat{\mathbf{x}}_{k}\right)^{2} / N} / M, i=1,2,3$.

Figure 1 shows the UBB noises in ellipsoid bound for process noises. First, observation noises with uniform distribution are generated. Then an ellipsoid is plotted according to the corresponding shape-defining matrix. The process noises which are contained in the ellipsoid are used as UBB disturbances.

After 100 Monte Carlo simulations, the averaged root mean square errors (RMSE) between true states and estimated states are shown in Figure 2. The point estimation accuracy of EEOB-SME is better than AESMF and KF. The RMSE of EEOB-SME for state $\mathbf{x}_{1}$ are larger than BA-SME at the initial stage but are gradual close to the BA-SME, which are the most time consuming algorithm. For state $\mathbf{x}_{2}$, the RMSE of EEOB-SME is equal to BA-SME and lower than AESMF and KF.

Plots in Figure 3 show averaged guaranteed bounds in each state variable. The bounds of EEOB-SME and BA-SME are close to AESMF for state $\mathbf{x}_{1}$ but are lower than AESMF for state $\mathbf{x}_{2}$. Notice that the bound of ellipsoids will not vanish and estimated results will not converge to single points with steps increasing.

Figure 4 shows the trends of scalar variable $\sigma^{2}$ with recursive updates. The scalar variable $\sigma^{2}$ of EEOB-SMF monotonous decreases exponentially from initial value 1 to values less than 0.1. The decline speed of EEOB-SME is faster than BA-SME, and the average run time of EEOB-SME is equal to AESMF. The shape-defining matrix $\sigma_{k}^{2} \mathbf{P}_{k}$ of ellipsoid will be reduced when the bounds of scalar variable $\sigma^{2}$ are decreasing. Notice that the smaller the value of scalar variable $\sigma^{2}$, the smaller the volume of ellipsoids, which can be seen from Figure 5. The values of scalar variable $\sigma^{2}$ in AESMF change a little after decreasing initial stage. 


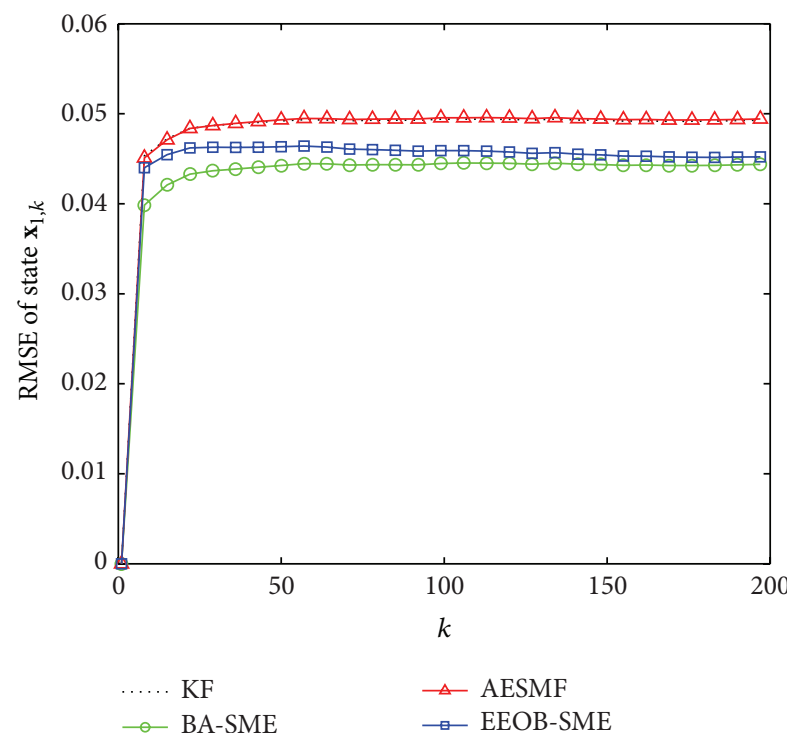

(a) State $\mathbf{x}_{1, k}$

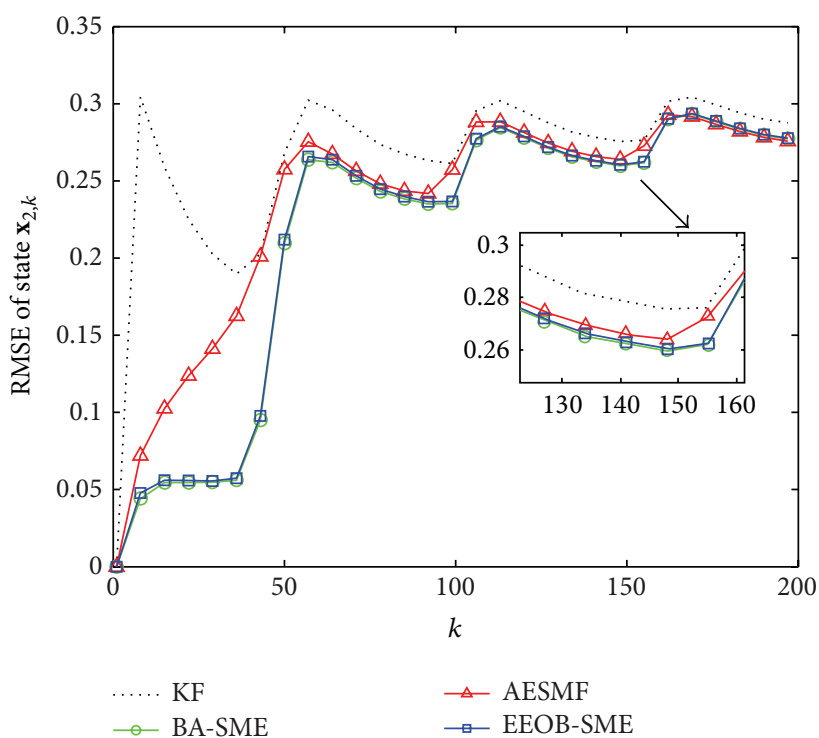

(b) State $\mathbf{x}_{2, k}$

FIGURE 2: Average RMS errors of states.

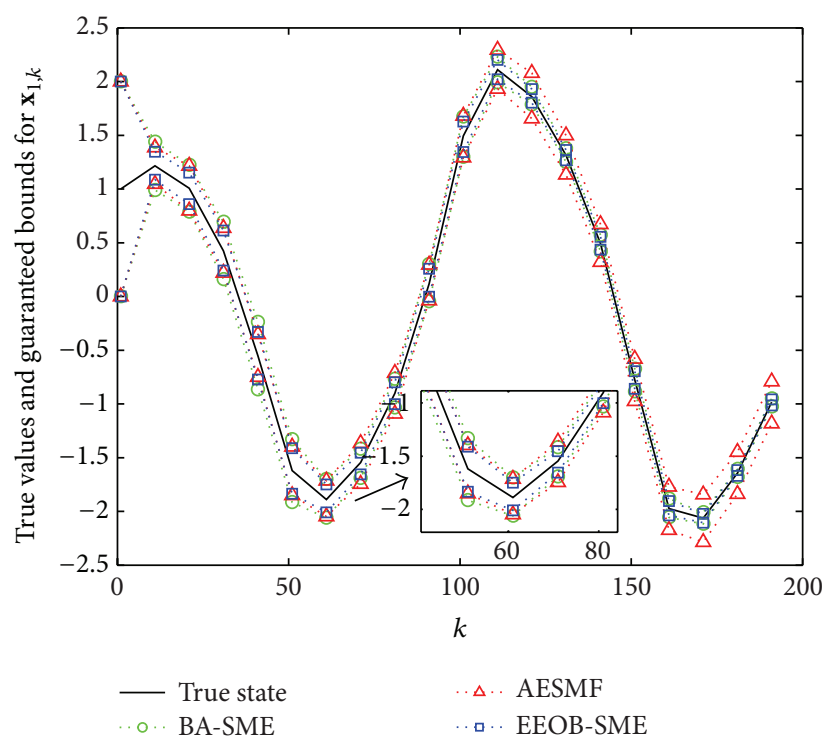

(a) State bounds $\mathbf{x}_{1, k}$

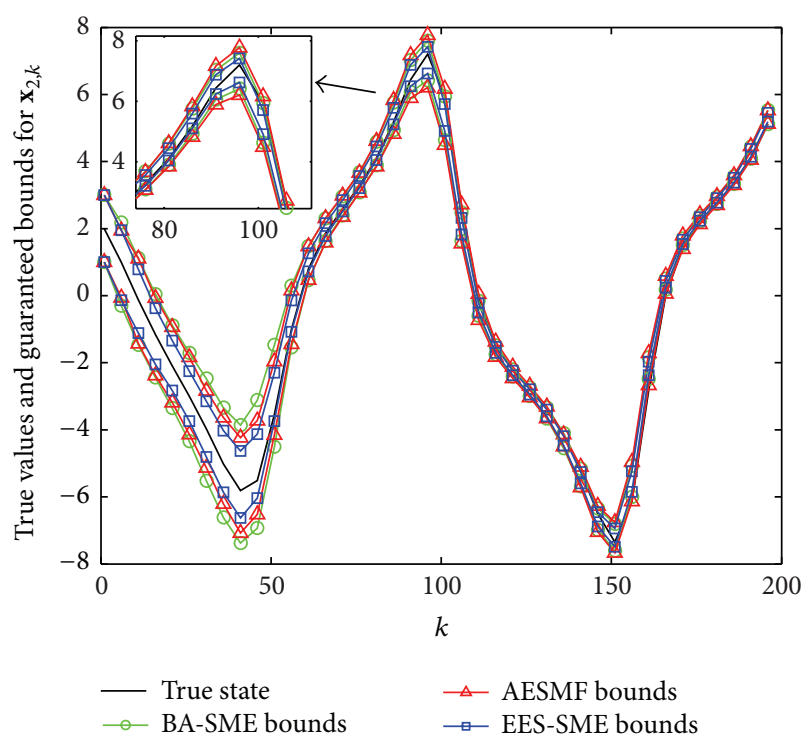

(b) State bounds $\mathbf{x}_{2, k}$

FIGURE 3: Guaranteed bounds and true values of states.

In Figure 5, states $\mathbf{x}_{1}$ are the horizontal axis and states $\mathbf{x}_{2}$ are the ordinate axis. As the direct result of remainder terms, the state uncertainty ellipsoid for EEOB-SME and AESMF algorithms decreases with recursive updates. Although ellipsoid feasible sets of EEOB-SME are larger than AESMF at the initial stage, with the decreasing of scalar variable $\sigma_{k}^{2}$, the volumes of ellipsoid feasible sets for EEOB-SME are equal to AESMF. Near the states $(-2,1)$, ellipsoid feasible sets of AESMF do not contain true states of the system, but the ellipsoid feasible sets of EEOB-SME always contain true states. Because of larger linearization errors, ellipsoid feasible sets of EEOB-SME and AESMF do not contain true states of nonlinear system, which can be seen at $k=100$.

The average RMSE and run time of EEOB-SME, BA-SME, AESMF, and Kalman Filter are summarized in Table 1. To pay at the cost of computing time, the RMSE of BA-SME algorithm is minimal. The average RMSE of EEOB-SME is larger than BA-SME but the computing time of EEOB-SME is lower than BA-SME. The most time consuming algorithm is $\mathrm{BA}-\mathrm{SME}$, which is more than $2 \mathrm{~ms}$ at each recursive step. The average rum time of EEOB-SME, AESMF, and Kalman Filter is less than $0.6 \mathrm{~ms}$ and suitable for real-time application. 


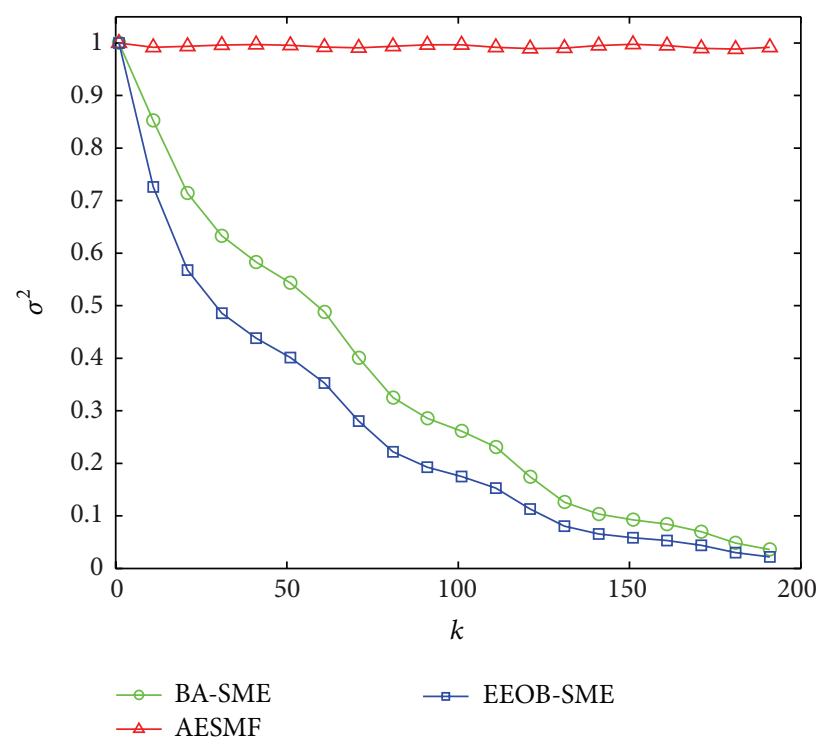

FIGURE 4: The scalar variable $\sigma_{k}^{2}$.

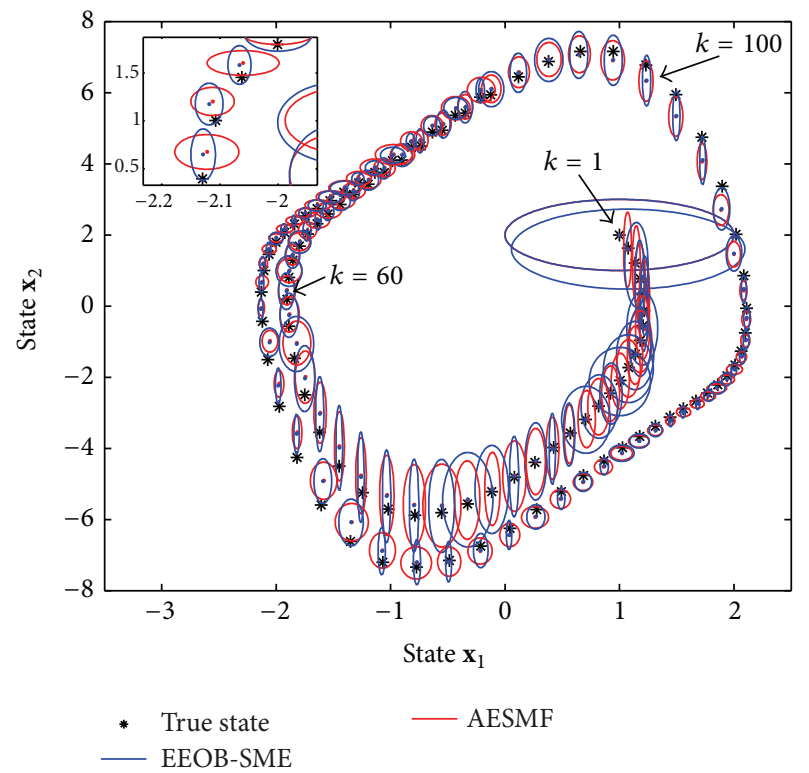

FIgURE 5: Bounding ellipsoids of the feasible sets.

TABLE 1: Average RMS errors of states and run time.

\begin{tabular}{lccc}
\hline \multirow{2}{*}{ Method } & \multicolumn{2}{c}{ RMSE } & Run time (ms) \\
& $e_{\mathrm{RMS}, 1}$ & $e_{\mathrm{RMS}, 2}$ & $t$ \\
\hline Kalman Filter & 0.0485 & 0.2708 & 0.224 \\
AESMF & 0.0481 & 0.2256 & 0.396 \\
BA-SME & 0.0436 & 0.2161 & 2.220 \\
EEOB-SME & 0.0453 & 0.2173 & 0.525 \\
\hline
\end{tabular}

\section{Conclusion}

Based on UBB disturbances, this paper proposes an EEOBSME algorithm for nonlinear discrete-time systems. The nonlinear equations of process and measurement equations are linearized with the first-order terms about current state estimations. Using interval analysis, the high order terms, which are equal to linearization errors, are bounded by ellipsoids. The bounds of the linearization errors are combined together with process or measurement disturbances as new UBB disturbances. If the nonlinear process and measurement equations are observable, the EEOB-SME algorithm is stable and the estimation errors of the EEOB-SME are bounded. Simulations results show that the EEOB-SME has better RMSE performance compared to Kalman Filter under UBB disturbances, and the computation time of EEOBSME is lower than BA-SME. In addition, the feasible sets of EEOB-SME contain more true states than AESMF. Thus, the real-time application of set-membership state estimation is improved by the EEOB-SME algorithms. Actually, the EEOBSME algorithm makes a balance between the estimation accuracy and computational efficiency. However, the EEOBSME does not acquire the minimal point estimation results, which is the future research direction.

\section{Competing Interests}

The authors declare that there are no competing interests regarding the publication of this paper.

\section{Acknowledgments}

This work has been supported in part by the National Natural Science Foundation of China under Grant no. 61233005 and National 973 Project under Grant no. 2014CB744200.

\section{References}

[1] R. E. Kalman, "A new approach to linear filtering and prediction problems," Journal of Basic Engineering, vol. 82, no. 1, pp. 35-45, 1960.

[2] A. H. Jazwinski, Stochastic Processes and Filtering Theory, Academic Press, New York, NY, USA, 1972.

[3] J. Hu, Z. Wang, H. Gao, and L. K. Stergioulas, "Extended Kalman filtering with stochastic nonlinearities and multiple missing measurements," Automatica, vol. 48, no. 9, pp. 20072015, 2012.

[4] S. Julier, J. Uhlmann, and H. F. Durrant-Whyte, "A new method for the nonlinear transformation of means and covariances in filters and estimators," IEEE Transactions on Automatic Control, vol. 45, no. 3, pp. 477-482, 2000.

[5] N. J. Gordon, D. J. Salmond, and A. F. M. Smith, "Novel approach to nonlinear/non-gaussian Bayesian state estimation," IEE Proceedings Part: F, vol. 140, no. 2, pp. 107-113, 1993.

[6] F. C. Schweppe, "Recursive state estimation: unknown but bounded errors and system inputs," IEEE Transactions on Automatic Control, vol. 13, no. 1, pp. 22-28, 1968.

[7] F. C. Schweppe, Uncertain Dynamic Systems, Prentice Hall, Englewood Cliffs, NJ, USA, 1973.

[8] L. Jaulin and E. Walter, "Set inversion via interval analysis for nonlinear bounded-error estimation," Automatica, vol. 29, no. 4, pp. 1053-1064, 1993.

[9] V. T. H. Le, C. Stoica, T. Alamo, E. F. Camacho, and D. Dumur, "Zonotopic guaranteed state estimation for uncertain systems," Automatica, vol. 49, no. 11, pp. 3418-3424, 2013. 
[10] D. P. Bertsekas and I. B. Rhodes, "Recursive state estimation for a set-membership description of uncertainty," IEEE Transactions on Automatic Control, vol. 16, pp. 117-128, 1971.

[11] E. Fogel and Y. F. Huang, "On the value of information in system identification-bounded noise case," Automatica, vol. 18, no. 2, pp. 229-238, 1982.

[12] F. L. Chernousko, State Estimation for Dynamic Systems, CRC Press, Boca Raton, Fla, USA, 1994.

[13] A. B. Kurzhanski, K. Sugimoto, and I. Valyi, "Guaranteed state estimation for dynamical systems: ellipsoidal techniques," International Journal of Adaptive Control and Signal Processing, vol. 8, no. 1, pp. 85-101, 1994.

[14] D. G. Maksarov and J. P. Norton, "State bounding with ellipsoidal set description of the uncertainty," International Journal of Control, vol. 65, no. 5, pp. 847-866, 1996.

[15] S. Dasgupta and Y.-F. Huang, "Asymptotically convergent modified recursive least-squares with data-dependent updating and forgetting factor for systems with bounded noise," IEEE Transactions on Information Theory, vol. 33, no. 3, pp. 383-392, 1987.

[16] S. Gollamudi, S. Nagaraj, S. Kapoor et al., "Set-membership state estimation with optimal bounding ellipsoids," in Proceedings of the International Symposium on Information Theory and Its Applications, pp. 262-265, Victoria, Canada, September 1996.

[17] G. Tan, C. Wen, and Y. C. Soh, "Identification for systems with bounded noise," IEEE Transactions on Automatic Control, vol. 42, no. 7, pp. 996-1001, 1997.

[18] C. Durieu, E. Walter, and B. Polyak, "Multi-input multi-output ellipsoidal state bounding," Journal of Optimization Theory and Applications, vol. 111, no. 2, pp. 273-303, 2001.

[19] Y. Becis-Aubry, M. Boutayeb, and M. Darouach, "State estimation in the presence of bounded disturbances," Automatica, vol. 44, no. 7, pp. 1867-1873, 2008.

[20] Y. Liu, Y. Zhao, and F. Wu, "Ellipsoidal state-bounding-based set-membership estimation for linear system with unknownbut-bounded disturbances," IET Control Theory \& Applications, vol. 10, no. 4, pp. 431-442, 2016.

[21] J. S. Shamma and K.-Y. Tu, "Approximate set-valued observers for nonlinear systems," IEEE Transactions on Automatic Control, vol. 42, no. 5, pp. 648-658, 1999.

[22] E. Scholte and M. E. Campbell, "A nonlinear set-membership filter for on-line applications," International Journal of Robust and Nonlinear Control, vol. 13, no. 15, pp. 1337-1358, 2003.

[23] B. Zhou, J. Han, and G. Liu, "A UD factorization-based nonlinear adaptive set-membership filter for ellipsoidal estimation," International Journal of Robust and Nonlinear Control, vol. 18, no. 16, pp. 1513-1531, 2008.

[24] T. Alamo, J. M. Bravo, M. J. Redondo, and E. F. Camacho, "A set-membership state estimation algorithm based on DC programming," Automatica, vol. 44, no. 1, pp. 216-224, 2008.

[25] F. Yang and Y. Li, "Set-membership fuzzy filtering for nonlinear discrete-time systems," IEEE Transactions on Systems, Man, and Cybernetics, Part B: Cybernetics, vol. 40, no. 1, pp. 116-124, 2010.

[26] M. E. Campbell, J.-W. Lee, E. Scholte, and D. Rathbun, "Simulation and flight test of autonomous aircraft estimation, planning, and control algorithms," Journal of Guidance, Control, and Dynamics, vol. 30, no. 6, pp. 1597-1609, 2007.

[27] W. Yu and J. de Jesús Rubio, "Recurrent neural networks training with stable bounding ellipsoid algorithm," IEEE Transactions on Neural Networks, vol. 20, no. 6, pp. 983-991, 2009.
[28] Y. Liu and Y. Zhao, "Ellipsoidal set filter combined setmembership and statistics uncertainties for bearing-only maneuvering target tracking," in Proceedings of the IEEE/ION Position, Location and Navigation Symposium (PLANS '14), pp. 753-759, IEEE, Monterey, Calif, USA, May 2014.

[29] X. Qing, F. Yang, and X. Wang, "Extended set-membership filter for power system dynamic state estimation," Electric Power Systems Research, vol. 99, pp. 56-63, 2013.

[30] R. E. Moore, R. B. Kearfott, and M. J. Cloud, Introduction to Interval Analysis, SIAM, Philadelphia, Pa, USA, 2009.

[31] K. Reif and R. Unbehauen, "The extended Kalman filter as an exponential observer for nonlinear systems," IEEE Transactions on Signal Processing, vol. 47, no. 8, pp. 2324-2328, 1999.

[32] Y. Song and J. W. Grizzle, "The extended Kalman filter as a local asymptotic observer for nonlinear discrete-time systems," Journal of Mathematical Systems, Estimation, and Control, vol. 5, no. 1, pp. 59-78, 1995.

[33] B. D. O. Anderson and J. B. Moore, "Detectability and stabilizability of time-varying discrete-time linear systems," SIAM Journal on Control and Optimization, vol. 19, no. 1, pp. 20-32, 1981. 


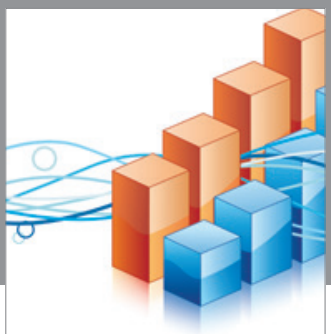

Advances in

Operations Research

vatem alat4

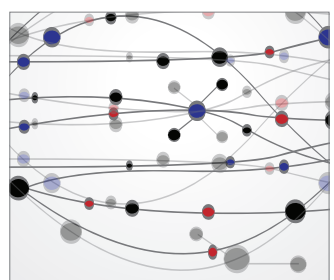

\section{The Scientific} World Journal
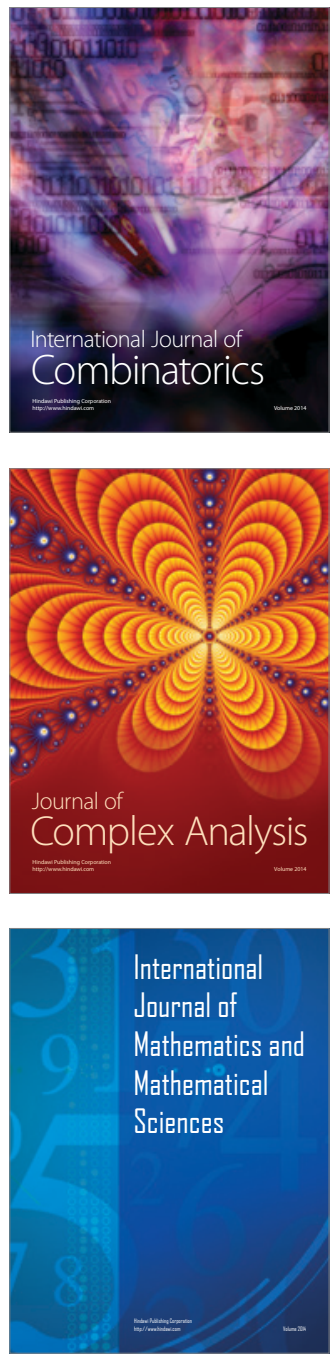
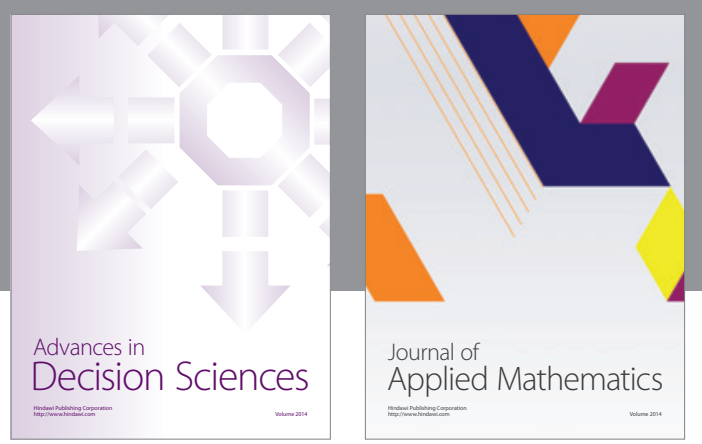

Algebra

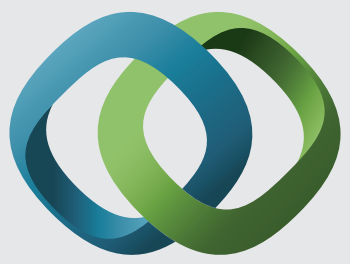

\section{Hindawi}

Submit your manuscripts at

http://www.hindawi.com
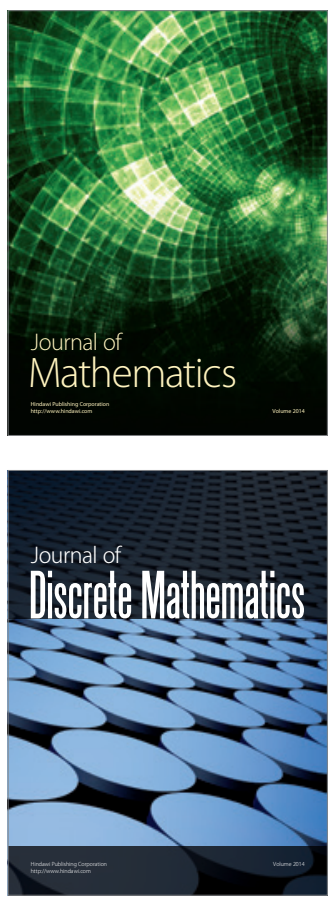

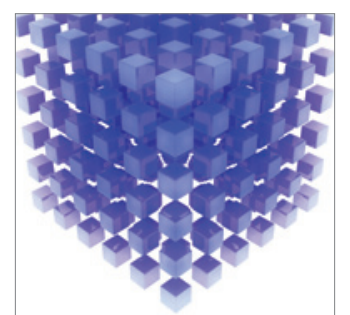

Mathematical Problems in Engineering
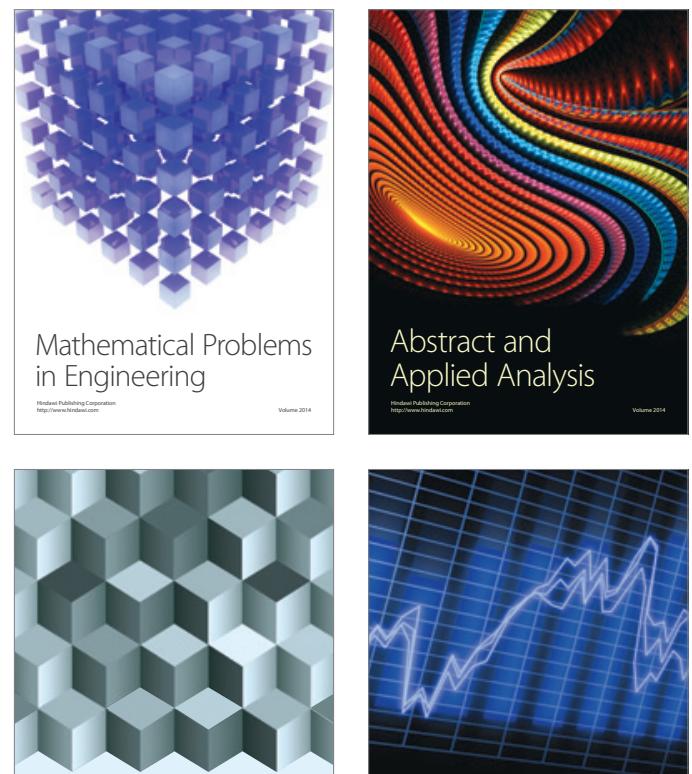

Journal of

Function Spaces

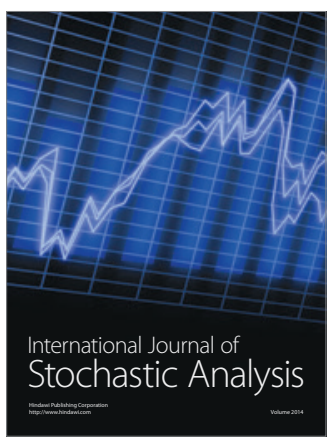

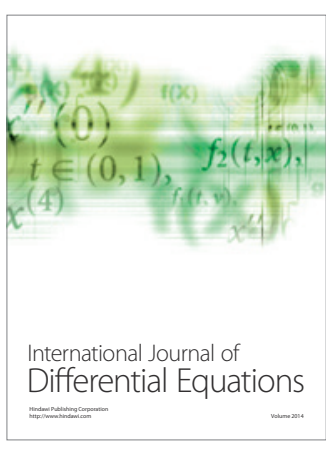
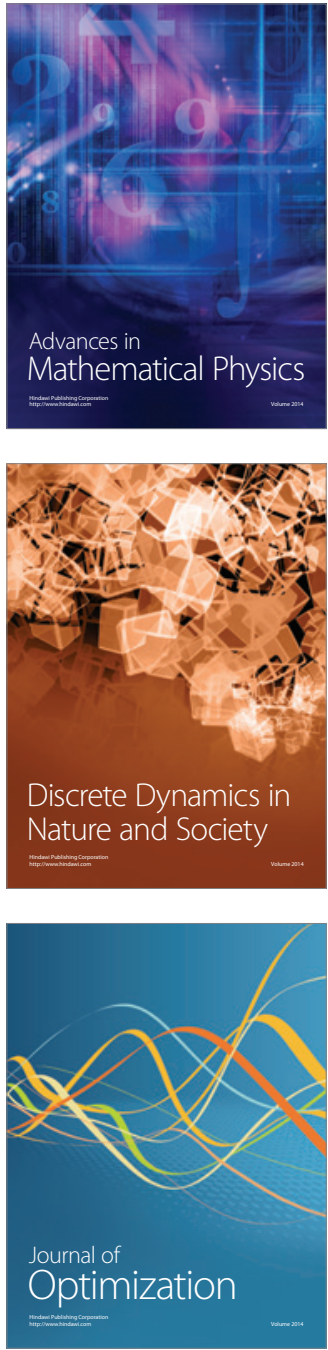